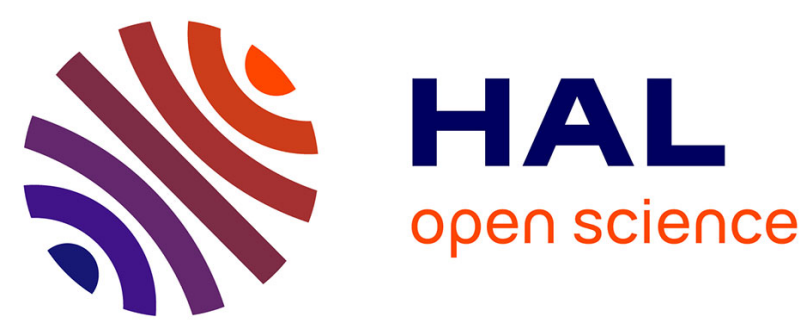

\title{
Lithographically Defined Cross-Linkable Top Coats for Nanomanufacturing with High- $\chi$ Block Copolymers
}

\author{
Xavier Chevalier, Cindy Gomes Correia, Gwenaelle Pound-Lana, Philippe \\ Bézard, Matthieu Sérégé, Camille Petit-Etienne, Guillaume Gay, Gilles \\ Cunge, Benjamin Cabannes-Boué, Célia Nicolet, et al.
}

\section{To cite this version:}

Xavier Chevalier, Cindy Gomes Correia, Gwenaelle Pound-Lana, Philippe Bézard, Matthieu Sérégé, et al.. Lithographically Defined Cross-Linkable Top Coats for Nanomanufacturing with High- $\chi$ Block Copolymers. ACS Applied Materials \& Interfaces, 2021, 13 (9), pp.11224-11236. 10.1021/acsami.1c00694 . hal-03218670

\section{HAL Id: hal-03218670 \\ https://hal.science/hal-03218670}

Submitted on 5 May 2021

HAL is a multi-disciplinary open access archive for the deposit and dissemination of scientific research documents, whether they are published or not. The documents may come from teaching and research institutions in France or abroad, or from public or private research centers.
L'archive ouverte pluridisciplinaire HAL, est destinée au dépôt et à la diffusion de documents scientifiques de niveau recherche, publiés ou non, émanant des établissements d'enseignement et de recherche français ou étrangers, des laboratoires publics ou privés. 


\title{
Lithographically Defined Cross-Linkable Top Coats for Nanomanufacturing with High- $\chi$ Block Copolymers
}

\author{
Xavier Chevalier,* Cindy Gomes Correia, Gwenaelle Pound-Lana, Philippe Bézard, Matthieu Sérégé, \\ Camille Petit-Etienne, Guillaume Gay, Gilles Cunge, Benjamin Cabannes-Boué, Célia Nicolet, \\ Christophe Navarro, Ian Cayrefourcq, Marcus Müller, Georges Hadziioannou, Ilias Iliopoulos, \\ Guillaume Fleury,* and Marc Zelsmann*
}

\begin{abstract}
The directed self-assembly (DSA) of block copolymers (BCPs) is a powerful method for the manufacture of high-resolution features. Critical issues remain to be addressed for successful implementation of DSA, such as dewetting and controlled orientation of BCP domains through physicochemical manipulations at the $\mathrm{BCP}$ interfaces, and the spatial positioning and registration of the BCP features. Here, we introduce novel top-coat (TC) materials designed to undergo cross-linking reactions triggered by thermal or photoactivation processes. The cross-linked TC layer with adjusted composition induces a mechanical confinement of the BCP layer, suppressing its dewetting while promoting perpendicular orientation of BCP domains. The selection of areas of interest with perpendicular features is performed directly on the patternable TC layer via a lithography step and leverages attractive integration pathways for the generation of locally controlled BCP patterns and nanostructured BCP multilayers.
\end{abstract}

KEYWORDS: lithography, block copolymers, self-assembly, cross-linkable top coats, top-coat patterning, nanopatterning, dewetting

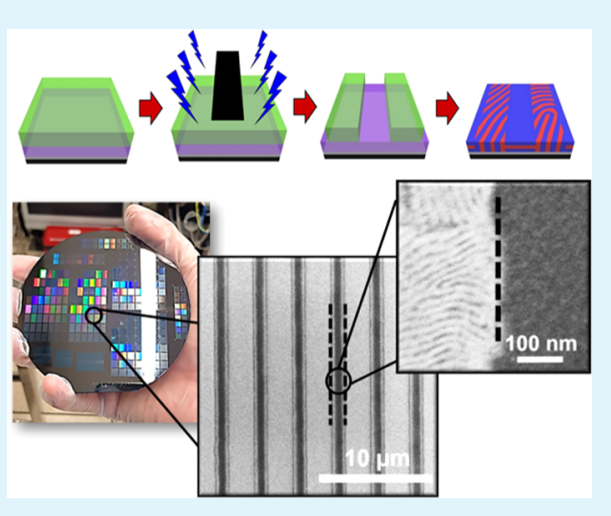

\section{INTRODUCTION}

Nanostructures formed by the self-assembly of block copolymers (BCPs) are foreseen to enable next-generation nanomanufacturing with applications in the design of functional materials and devices in biology, optics, or microelectronics. Further opportunities in the development of BCP nanotechnologies are tied to the formation of well-ordered periodic nanoscale patterns in thin-film configuration. ${ }^{1-5}$ Indeed, the thermodynamic driving force leading to the segregation of BCP chains in the form of chemically dissimilar nanodomains ${ }^{6,7}$ can yield a mask further used to define features in microelectronic applications. ${ }^{3,8,9}$ Extensive work has been pursued over the years to enable the directed selfassembly (DSA) of BCPs with potent advances both in the methods to perpendicularly orient $\mathrm{BCP}$ domains ${ }^{10}$ and in the precise registration of the $\mathrm{BCP}$ nanostructures with the fabrication of guiding templates, relying on chemoepitaxy and graphoepitaxy. ${ }^{11,12}$

With the advent of BCP systems allowing the definition of sub-10 nm features (the so-called "high- $\chi$ BCPs", where $\chi$ is the Flory-Huggins parameter), ${ }^{13}$ interface manipulations to provide neutral conditions at both interfaces of the BCP layer have become a critical aspect of BCP lithography. Following the pioneering work of Bates et al. on polarity switching top coats (TCs) for the controlled orientation of silicon-containing
BCPs, ${ }^{14,15}$ Suh et al. have recently demonstrated how a crosslinked layer deposited by chemical vapor deposition can be applied to the fabrication of orientation-controlled BCP structures. ${ }^{16}$ However, in this latter case, the fully organic BCP necessitates time-consuming TC deposition process and extended infiltration procedures for pattern transfer, impeding thus high-throughput of wafers for microelectronic industry. Surface treatment of BCP films with plasma has also been shown to efficiently produce perpendicular BCP features, as the resulting structural amorphization of a thin BCP layer at its top surface is conceptually similar to a grafted brush layer of statistical copolymers. ${ }^{17,18}$ Besides, a self-assembled BCP film located on top of an underlying high- $\chi$ BCP layer can also provide an efficient orientational control of high- $\chi$ BCP domains when the surface energies between the two layers are carefully matched. ${ }^{19,20}$ Nevertheless, this latter concept was demonstrated only for fully organic high- $\chi$ BCPs with large periods, although smart layering processes were employed to

Received: January 12, 2021

Accepted: February 16, 2021

Published: February 23, 2021

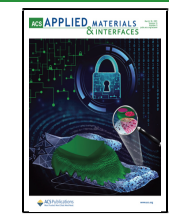



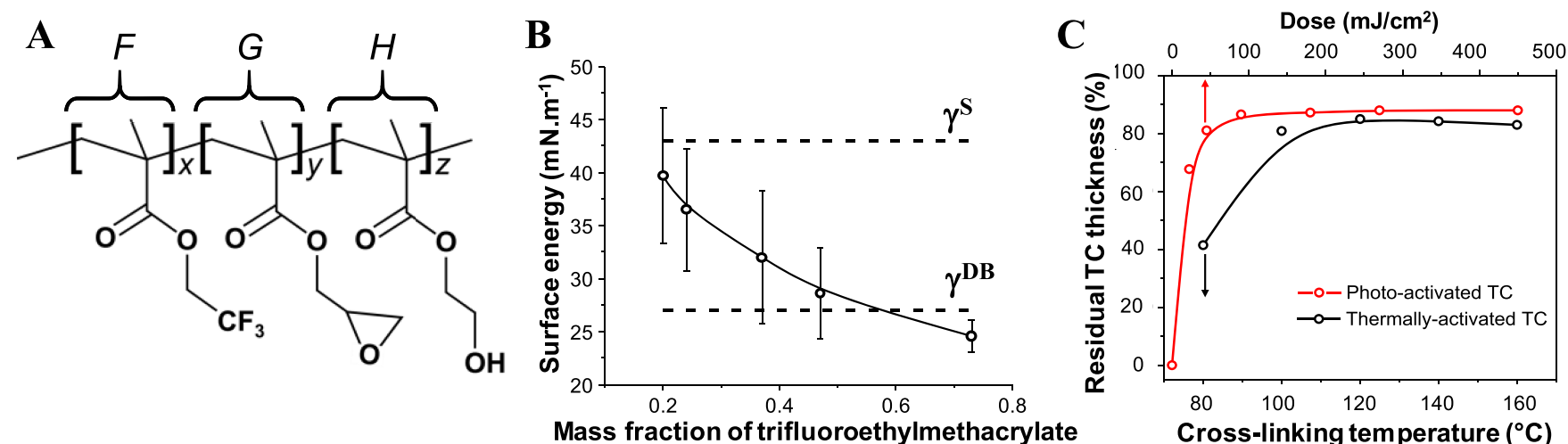

Figure 1. (A) Macromolecular architecture of the FGH terpolymer enabling balanced interfacial energy with BCP domains, dewetting suppression of $\mathrm{BCP}$ layers, and novel integration schemes via thermal activation or photoactivation. $x, y$, and $z$ denote the degree of polymerization of each unit in the terpolymer. (B) Effective variation of the surface energy of the FGH terpolymer materials cross-linked with ammonium triflate as ITC at 90 ${ }^{\circ} \mathrm{C}$ during $3 \mathrm{~min}$ as a function of the mass fraction of 2,2,2-trifluoroethylmethacrylate (see Tables 1 and S2 for detailed macromolecular characteristics and surface energy measurements, respectively) with black line as a guide to the eye. The dotted lines correspond to the surface tension of poly(1,1-dimethyl silacyclobutane) "DB" and poly(styrene) "S" ( $\gamma^{\mathrm{DB}}$ and $\gamma^{\mathrm{S}}$ respectively). (C) Thermal activation and photoactivation efficiency of the TC cross-linking evaluated as the residual TC thickness after development in alcoholic solvents with the black and red lines as guides to the eye (FGH-37 was used for this particular experiment with an initial thickness of $50 \mathrm{~nm}$ ). Using ammonium triflate as ITC, a constant residual thickness is obtained by thermal annealing at $100{ }^{\circ} \mathrm{C}$ for $2 \mathrm{~min}$. Using (4-phenylthiophenyl)diphenylsulfonium triflate as PAG, a $100 \mathrm{~mJ}$. $\mathrm{cm}^{-2}$ exposure dose leads to a constant TC thickness after the development step in alcoholic solvents.

achieve perpendicular features. It is noteworthy that early attempts to pattern BCP layers self-assembled onto chemical templates through a poly(methyl methacrylate) top coat were pursued by Yoshida et al. ${ }^{21}$ However, in this particular study, the e-beam lithographic step is rather employed to remove the TC layer instead of an actual patterning. In addition, the poly(methyl methacrylate) TC cannot be directly spin-coated onto the BCP layer and suffers from low sensitivity toward ebeam exposure, mitigating the interest of such approach.

Despite these significant breakthroughs, several fundamental and technical challenges remain to be addressed in order to fulfill crucial industrial requirements over the whole DSA process. For instance, nonuniform coating of the TC layer, pronounced dewetting of the $\mathrm{BCP}$ layer during thermal annealing, $^{22-24}$ or the selection of areas of interest in the templated BCP assembly needed for integrated circuit design $^{25,26}$ are potentially severe limitations for further nanomanufacturing by DSA of BCPs. More precisely, highly relevant challenges such as the suppression of BCP dewetting and the precise selection of areas of interest through direct patterning of the top coat have never been addressed in the literature, even though of crucial importance for the implementation of BCP nanomanufacturing.

Here, we introduce an innovative thermally activated and photoactivated TC design that leverages the controlled orientation of BCP nanostructures in thin film configuration while successfully addressing all the aforementioned bottlenecks and challenges owing to the on-demand cross-linking of these interfacial layers. Additionally, this strategy facilitates multiple integration schemes for the development of complex three-dimensional nanostructures enabled by the unique TC patterning properties via common lithographic tools.

\section{RESULTS AND DISCUSSION}

\subsection{Design of Thermally- and Photocross-Linkable} Top Coats. Our approach relies on readily spin-casted thermally activated or photoactivated TCs to control the dewetting and the orientation of nanostructured BCP thin films while allowing novel integration pathways and increased opportunities for applications in DSA. The TC chemical platform is based on a statistical terpolymer architecture of poly (2,2,2-trifluoroethylmethacrylate-stat-glycidylmethacrylate-stat-2-hydroxyethylmethacrylate) (abbreviated FGH- $x$, where $x$ is the mass fraction of 2,2,2-trifluoroethylmethacrylate expressed as a percentage). This platform provides both a fine control of the interfacial energy between the BCP domains and the TC layer, that is, through optimization of the terpolymer composition, as well as thermally activated or photoactivated cross-linking capabilities via the pendant epoxy moieties (Figure 1A,B and Table 1). The straightforward synthesis of

Table 1. Macromolecular Characteristics of the FGH Terpolymers

$\begin{array}{cccccc}\text { TC materials } & f_{\text {GMA }}{ }^{a} & f_{\text {HEMA }}{ }^{a} & f_{\text {MATRIFE }}{ }^{a} & M_{\mathrm{n}}\left(\mathrm{kg} \cdot \mathrm{mol}^{-1}\right)^{b} & D^{b} \\ \text { FGH-20 } & 0.19 & 0.61 & 0.20 & 2.1 & 1.6 \\ \text { FGH-24 } & 0.2 & 0.56 & 0.24 & 3.05 & 1.7 \\ \text { FGH-37 } & 0.26 & 0.37 & 0.37 & 2.7 & 1.9 \\ \text { FGH-47 } & 0.27 & 0.26 & 0.47 & 2.8 & 1.9 \\ \text { FGH-73 } & 0.27 & 0 & 0.73 & 2.8 & 1.9\end{array}$

${ }^{a}$ Determined by ${ }^{1} \mathrm{H}$ NMR in $\mathrm{CDCl}_{3}$. ${ }^{b}$ Determined by SEC using THF as eluent at $40{ }^{\circ} \mathrm{C}$ with poly(styrene) standards.

the FGH materials associated with the commercial availability of their constitutive monomers allows a facile tuning of their interfacial properties via their relative chemical composition. It confers a substantial flexibility to the system for its adaptation to different $\mathrm{BCP}$ systems by enabling the crucial design of neutral and affine TCs (N-TC and A-TC, respectively) mandatory for particular integration schemes (see "Novel integration schemes" section). It is noteworthy that the FGH materials can also be used as grafted or cross-linked neutral underlayers (NLs), owing to their multifunctional architecture.

The activation of the cross-linking process is triggered on demand either by a latent ionic thermal catalyst (ITC) or photoacid generator (PAG) incorporated in the TC solution. The TC material is formulated in common solvents orthogonal to the BCP films (i.e., alcoholic solvents in our case) of 


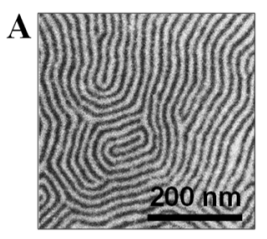

B
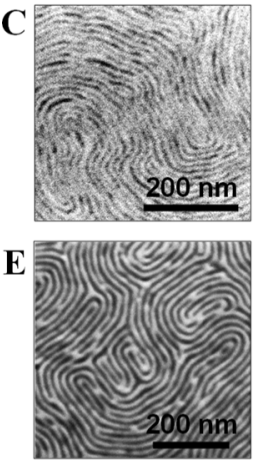

D

F
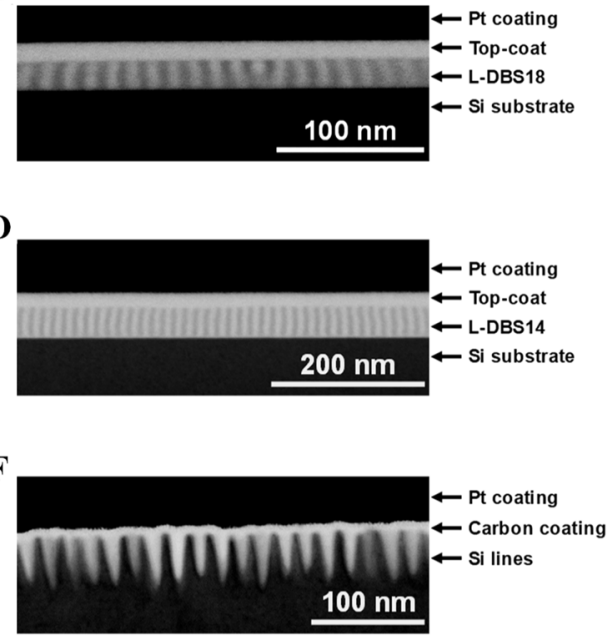

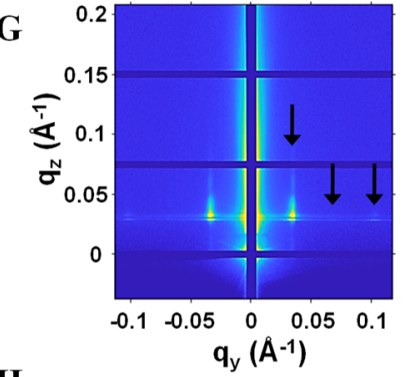

$\mathbf{H}$

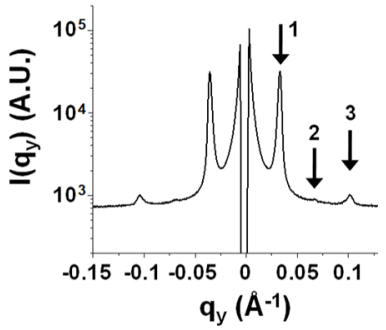

Figure 2. (A-D) Controlled out-of-plane orientation of lamellar nanostructured DBS thin films obtained using cross-linked TCs. Top-view SEM and cross-sectional FIB-STEM images of (A,B) $30 \mathrm{~nm}$ thick L-DBS18 film and (C,D) $50 \mathrm{~nm}$ thick L-DBS14 film, respectively. The cross-linking of the FGH-37 TC layers (clearly visible on the FIB-STEM images) was activated through thermal annealing at $90{ }^{\circ} \mathrm{C}$ for 3 min, followed by the promotion of the self-assembly of the L-DBS18 and L-DBS14 layers at $240{ }^{\circ} \mathrm{C}$ for $5 \mathrm{~min}$. The TC layer was etched away with an $\mathrm{Ar} / \mathrm{O}_{2}$-based plasma before SEM characterization. The dark and bright domains in the FIB-STEM images correspond to the DB and S phase, respectively. (E,F) Top-view SEM and cross-sectional FIB-STEM images of L-DBS18 mask transferred into the Si substrate using a three-step plasma process. This process produces aspect-ratio features of around 5 , with depth $\approx 45 \mathrm{~nm}$ and critical dimension $\approx 9 \mathrm{~nm}$. (G,H) 2D GISAXS pattern and GISAXS intensity cut along the horizontal $q_{y}$ direction taken at $q_{z}=0.03 \mathrm{~A}^{-1}$. The black arrows on the 2D GISAXS pattern point to the observed Bragg rods with a series of peaks at scattering wave vectors indexed as $q_{y} / q_{y}{ }^{*}=1,2,3$ on the intensity cut along the horizontal $q_{y}$ direction.

appropriate surface tensions with respect to the BCP components, thus providing an excellent coating quality over the whole wafer surface (Figure S1). Figure 1C shows the efficiency of the cross-linking reaction evaluated on a bare $\mathrm{Si}$ substrate as the residual thickness of the layers obtained after a development step in alcohols. Both thermally activated and photoactivated TC layers undergo efficient cross-linking in a timeframe of a few minutes, compatible with the semiconductor industry processes. Interestingly, the cross-linking process does not only preserve the TC uniformity by suppressing its viscous flow but it also provides a strong mechanical confinement owing to its own high flexural rigidity, thus offsetting the BCP dewetting prone to appear for such thin films during the thermal annealing step (see next section for further details on dewetting). Additionally, the intrinsic rigidity of the cross-linked TC layer suppresses the formation of holes and islands and thereby provides a further thermodynamic driving force to stabilize the perpendicular orientation of the BCP domains (see Supplementary Note S1).

Such a TC concept with tunable surface energy enables the controlled orientation of BCP domains for systems insoluble in polar alcoholic solvents, provided that the neutrality condition is guaranteed at some FGH composition. ${ }^{27}$ As a demonstration, it was applied to a poly(1,1-dimethyl silacyclobutane)block-poly(styrene) silicon-containing BCP system (denoted "DBSp" where $p$ is the natural periodicity of the BCP determined by small-angle X-ray scattering, and the prefix " $\mathrm{L}$ " or "C" indicates a lamellar or cylindrical morphology, respectively) with periodicity as low as $12 \mathrm{~nm}$ (Table $S 1$ and Figure S3). For this BCP system, coated on either substrategrafted or cross-linked FGH materials, the compositional effect of the different FGH architectures used as TCs and underlayers is summarized on the self-assembly cartographies shown in Figure S2. It is worth mentioning that a neutral TC is found for FGH composition(s) of intrinsic surface energy between that of the BCP blocks. Additionally, both neutral and affine TC configurations can be found, thus enabling dedicated integration schemes (see "Novel integration schemes" section). Figure 2 illustrates a neutral configuration, where the BCP of arbitrary thickness is sandwiched between the NL and the thermally cross-linked neutral TC (FGH-37). After thermal annealing $\left(240{ }^{\circ} \mathrm{C}, 5 \mathrm{~min}\right)$, an out-of-plane lamellar structure is obtained for both L-DBS18 and L-DBS14 BCPs. No indications of terraced structures related to in-plane lamellae were observed by either scanning electron microscopy (SEM) or scanning transmission electron microscopy (STEM) imaging, despite the large difference in surface energies $(\gamma)$ between poly(1,1-dimethyl silacyclobutane) $\left(\gamma^{\mathrm{DB}} \approx 27 \mathrm{mN}\right.$. $\left.\mathrm{m}^{-1}\right)$ and poly(styrene $)\left(\gamma^{\mathrm{S}} \approx 43 \mathrm{mN} \cdot \mathrm{m}^{-1}\right)$. Additional grazing incidence small-angle X-ray scattering (GISAXS) measurements for L-DBS18 were performed to probe the out-of-plane orientation. The corresponding results further confirmed the conclusions obtained from STEM imaging. The 2D GISAXS patterns feature Bragg rods along the $q_{z}$ direction with a series of peaks at scattering wave vectors indexed as $q_{y} / q_{y}{ }^{*}=1,2,3$, where $q_{y}{ }^{*}$ is the position of the first-order Bragg rod (Figure $2 \mathrm{G}, \mathrm{H})$. The out-of-plane lamellae for L-DBS18 were subsequently transferred into the $\mathrm{Si}$ substrate in a three-step process. First, a $\mathrm{H}_{2} / \mathrm{N}_{2}$ RIE plasma treatment is performed to remove the TC layer, followed by selective removal of the $S$ phase with a $\mathrm{N}_{2} / \mathrm{O}_{2}$ RIE plasma, and the remaining $\mathrm{DB}$ lamellae are eventually pattern-transferred with a $\mathrm{HBr} / \mathrm{O}_{2}$ synchronously pulsed inductively coupled plasma (ICP) plasma $^{28,29}$ with a high mask fidelity (Figure 2E,F). This process produces high aspect ratio features with a line width of $9 \mathrm{~nm}$ and a depth of $45 \mathrm{~nm}$. Such characteristics for the substrate-transferred features alleviate the constraint of thin- 


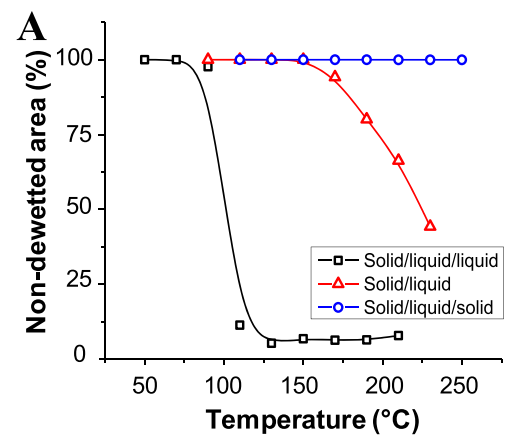

B
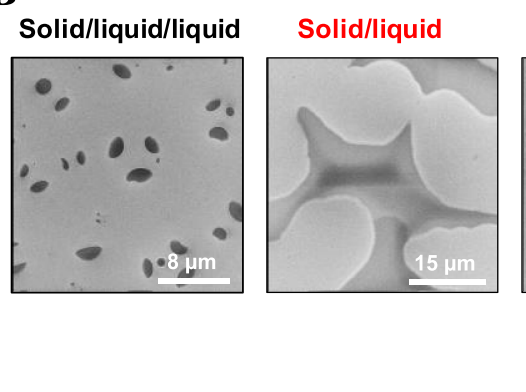

Solid/liquid/solid

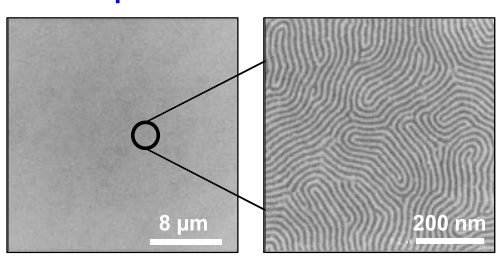

Figure 3. (A) Detailed dewetting map of a $30 \mathrm{~nm}$ thick L-DBS18 layer coated on a NL grafted onto the Si substrate and covered by a thermallyactivated $50 \mathrm{~nm}$ thick FGH-37 layer as a function of the annealing temperature $(5 \mathrm{~min})$ : (black squares) "solid/liquid/liquid" configuration, (red triangles) "solid/liquid" configuration, and (blue circles) "solid/liquid/solid" configuration. Each data point represents the mean area coverage of seven random SEM images on a specific sample (one sample per temperature increment). (B) SEM pictures showing the extent of dewetting for each configuration at the highest temperature reported in (A) dark gray areas correspond to thicker regions. In the SEM image exhibiting phaseseparated features, light-gray areas correspond to the poly(1,1-dimethyl silacyclobutane) phase of the L-DBS18 BCP.

ness for sacrificial Si-based layers into actual materials stack to allow in fine higher aspect-ratio functional features.

2.2. Dewetting Suppression. A critical aspect of BCP lithography is related to dewetting phenomena occurring during the promotion of BCP self-assembly. Despite pioneering works performed on the dewetting of polymeric thin films, ${ }^{30,31}$ this particular topic remains rather unexplored in DSA lithography ${ }^{23,24,32}$ even though uniform BCP coating is critical for industrial implementation. Corresponding research studies only exemplify the smart use of dewetted BCP features to obtain patterns of potential interest, but none of them deals with its suppression. It must be noted here that even for the "simple" homopolymer case, the proposed methods aiming to suppress dewetting or slow-down its kinetic are extremely limited, and none of them are transposable to BCPs in the framework of DSA lithography. ${ }^{33}$ It is therefore of crucial importance to develop materials and processes enabling the generation of highly uniform BCP film for nanomanufacturing with DSA, especially in the case of high- $\chi$ BCP systems. Indeed, the deleterious effects of dewetting are particularly pronounced for BCPs with low periods as both, the high molecular diffusivity and the thin film configuration (typically below $50 \mathrm{~nm}$ ) of the BCP layer, promote the dewetting kinetics. Additionally, the wetting behavior of the BCP layer is impacted by the large surface free-energy difference between the BCP blocks (a typical property of high- $\chi$ BCPs) as a situation providing simultaneous wetting of both blocks on a common substrate is intricate. Moreover, the grafted NLs used to balance the interfacial interactions between the substrate and the BCP domains further accentuates the BCP dewetting due to both entropic and enthalpic penalties for the BCP chains to interdigitate into the NL layer for realistic lithography-dedicated configurations (see Supplementary Note S1 for an in-depth discussion). Accordingly, the wetting of a BCP layer coated on a NL is typically a thermodynamically unstable situation, that is, the system aims to evolve toward a dewetted configuration. Experimentally, a trade-off between the promotion of the BCP self-assembly and the minimization of BCP dewetting is therefore often required, limiting the longrange ordering of the self-assembled features as a result by increasing the probability of defects such as disclinations and edge dislocations. $^{34-36}$

The thermally activated and photoactivated TCs developed in this study play a key role in counteracting such intrinsic dewetting. The cross-linking of the TC layer yields an increase in its own flexural rigidity, as attested by the observed increase in its Young modulus measured by atomic force microscopy (AFM) nanoindentation (Figure S4), resulting in a stiff layer, which subsequently inhibits the distortion of the BCP layer planarity. The thickness of the spin-coated FGH TC material plays here an important role. Indeed, the flexural rigidity, $D$, of a flat layer is directly proportional to the product between its Young modulus, $E$, and cubed thickness, $h^{3}$. Accordingly, a thicker TC layer is more prone to suppress the dewetting of the underlying viscoelastic BCP layer. In the case of the TCs examined in this study, preliminary experiments indicated that a $20 \mathrm{~nm}$ FGH thickness is sufficient for an efficient suppression of BCP dewetting. The effect of the strong mechanical confinement induced by the cross-linking step on the suppression of the dewetting for the viscoelastic BCP layer was thoroughly demonstrated for both the thermally and photoactivated TC layers using a $50 \mathrm{~nm}$ TC thickness. In the following, unless stated otherwise, this particular TC thickness was used as a standard, mainly owing to its relevance as regard to the thicknesses of standard lithographic resists. In the case of thermally cross-linked TC, we applied first a 3 min bake at $90{ }^{\circ} \mathrm{C}$ to initiate the cross-linking reaction, then a $5 \mathrm{~min}$ thermal solicitation at various temperatures, and probed the BCP dewetting through SEM image analysis. Figure 3A compares the dewetting characteristics of a NL/L-DBS18/TC stack (a "solid/liquid/solid" configuration) with a thermally cross-linked TC to two reference configurations: (i) a "solid/ liquid" configuration, that is, a L-DBS layer ("liquid") coated on its neutral layer ("solid") without any TC and (ii) a "solid/ liquid/liquid" configuration, that is, a L-DBS layer coated on its neutral layer with a non-cross-linked TC ("liquid"). The onset of dewetting is located around 170 and $90{ }^{\circ} \mathrm{C}$ for the "solid/liquid" and "solid/liquid/liquid" configurations, respectively. In this latter case, the viscous flow inherent to the low glass transition temperature of the TC layer $\left(T_{\mathrm{g}} \approx 20{ }^{\circ} \mathrm{C}\right.$, due to low molecular weight TCs) is directly responsible for the lower temperature observed for the onset of dewetting kinetics, ${ }^{37}$ highlighting the limitations of a "solid/liquid/ liquid” configuration in a BCP lithography process using TCs.

The TC cross-linking, leading to a "solid/liquid/solid" configuration, was performed with an ITC at a temperature for which the DBS is still in a solid state (below both the $T_{\mathrm{g}}$ of the poly(styrene) block and the order-disorder transition temper- 

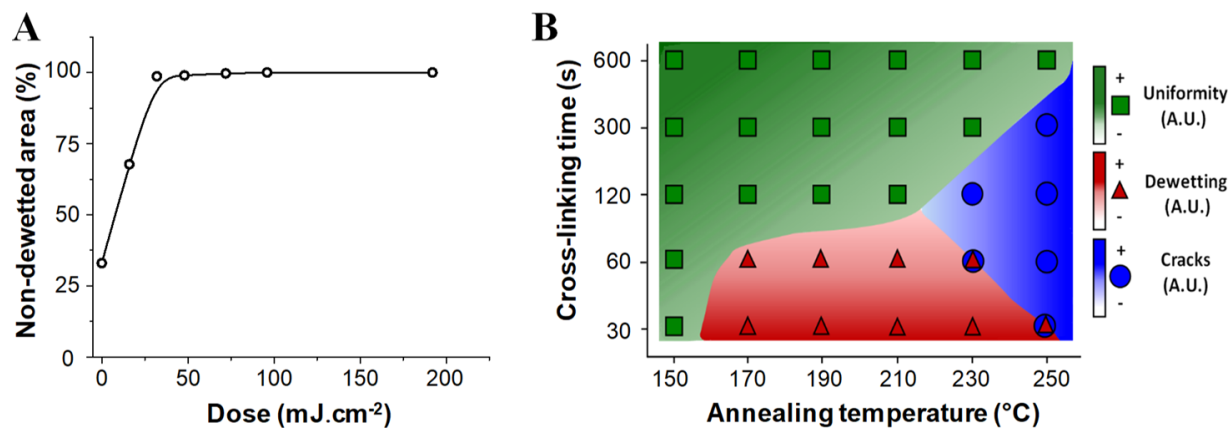

Figure 4. (A) Detailed dewetting map of a $30 \mathrm{~nm}$ thick L-DBS18 layer coated on a NL grafted onto the Si substrate and covered by a photoactivated $50 \mathrm{~nm}$ thick FGH-37 layer as a function of the applied UV dose $(\lambda=365 \mathrm{~nm})$ and subsequent thermal annealing at $260{ }^{\circ} \mathrm{C}$ for 5 min. Each data point represents the mean area coverage of seven random SEM images on a specific sample (one sample per applied UV dose). (B) Detailed map of mechanical stress configurations in the referential cross-linking time/annealing temperature ( 5 min). The cross-linking temperature of the TC was fixed at $90{ }^{\circ} \mathrm{C}$. Optimized experimental parameters (green area) yield uniform BCP films without observable dewetting (red area) or cracks (blue area). The color scale gradients indicate the probability of an event and are provided as a guide to the eye.
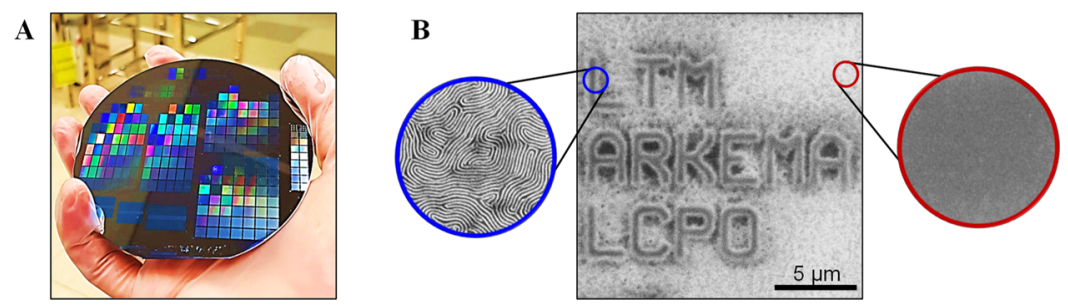

Figure 5. (A) 4-in. Si wafer with a NL/L-DBS18/TC stack. The TC patterning was performed with UV-365 nm lithography. The iridescent colors are due to light diffraction arising from TC gratings with different dimensions. (B) Proof of concept of the TC lithography illustrated with electronbeam lithography as the patterning tool. The TC patterned areas (i.e., the logos) subsequently drive the formation of perpendicular BCP features, while the remaining BCP film consists of parallel features. The TC is readily hashed at the end of the process to reveal the orientational changes in the BCP film related to the TC patterning step.

ature), that is, at $90{ }^{\circ} \mathrm{C}$, to avoid an early dewetting. In contrast to the "solid/liquid/liquid" and "solid/liquid" configurations, $100 \%$ of the inspected area is covered by the BCP layer after the thermal solicitation for the whole set of applied temperatures (Figure 3A,B). Indeed, the TC cure prevents the amplification of capillary waves ${ }^{37}$ in the BCP layer owing to the imposed mechanical confinement. The thickness fluctuations in the BCP layer are thus restrained, while the capillary forces ensure the cohesion of the NL/BCP/TC stack. Consequently, the dewetting of the BCP is readily inhibited, even at elevated annealing temperatures, while a proper phaseseparation behavior is retained at the nanoscale. This was further corroborated using a rapid thermal annealing tool for the self-assembly, ${ }^{38,39}$ for which temperatures up to $350{ }^{\circ} \mathrm{C}$ can be applied to the cured NL/L-DBS18/TC stack without any dewetting (Figure S5). The introduction of an elastic force inherent to the formation of a rubbery cross-linked network has been shown to inhibit both the dewetting and the relaxation of residual stresses in the corresponding spin-casted layers. $^{40-42}$ Its impact regarding the dewetting of a bilayer presenting a viscoelastic underlayer was demonstrated to slow down the dewetting kinetics due to competing mechanisms of the cross-linking and dewetting processes, leading thus to a final frozen state with a reduction of the nucleated hole density and their maximum size. ${ }^{43}$ In contrast, here, we clearly demonstrate that the dewetting of the underlayer is entirely suppressed if the cross-linking reaction proceeds in a shorter time frame than the dewetting nucleation time, yielding a uniform flat stack.

The cross-linking of the TCs can be also activated using PAGs, which are more relevant to the semiconductor industry, as the FGH epoxy-methacrylate platform behaves as standard chemically amplified negative-tone lithography resists. ${ }^{44} \mathrm{Be}$ sides, the catalytic proton is available at room temperature, further reducing potential thermally activated dewetting during the cure while allowing rapid cross-linking of the TC layer at low dose (even for a PAG activation at $365 \mathrm{~nm}$ with low quantum yields). ${ }^{45}$ As demonstrated in Figure $4 \mathrm{~A}$, the dewetting of a L-DBS18 film, thermally annealed at $260{ }^{\circ} \mathrm{C}$, is entirely suppressed for a UV dose as low as $30 \mathrm{~mJ} \cdot \mathrm{cm}^{-2}$.

It is noteworthy that the mismatch of thermal expansion coefficients (TEC) between the different layers (i.e., a lower TEC for the cross-linked TC and a higher TEC for the DBS) induces mechanical stresses prone to generate cracks during the thermal annealing at elevated temperatures. As highlighted in Figure 4B, an optimization of the process can easily circumvent this issue through the control of the TC crosslinking degree, while keeping in mind that the self-assembly budget has to be maximized in the framework of defect reduction for DSA. ${ }^{46,47}$ The cross-linking has to produce a material stiff enough to inhibit the dewetting of the BCP but ductile enough to self-adapt to the expansion of the BCP layer at the annealing temperature. Interestingly the potential formation of wrinkles in the TC layer prone to appear during thermal treatment was not observed. We strongly believe that the exceptional mechanical strength of the FGH TC layer inhibits the wrinkling mechanism inherent to stress relaxation due to a favorable ratio between the bending stiffness of the TC "skin" layer, $B$, and an effective elastic foundation of stiffness $K$, representative of the BCP "viscoelastic" underlayer. ${ }^{48}$ Therefore the FGH materials and the associated crosslinking processes are not only able to suppress the dewetting of 

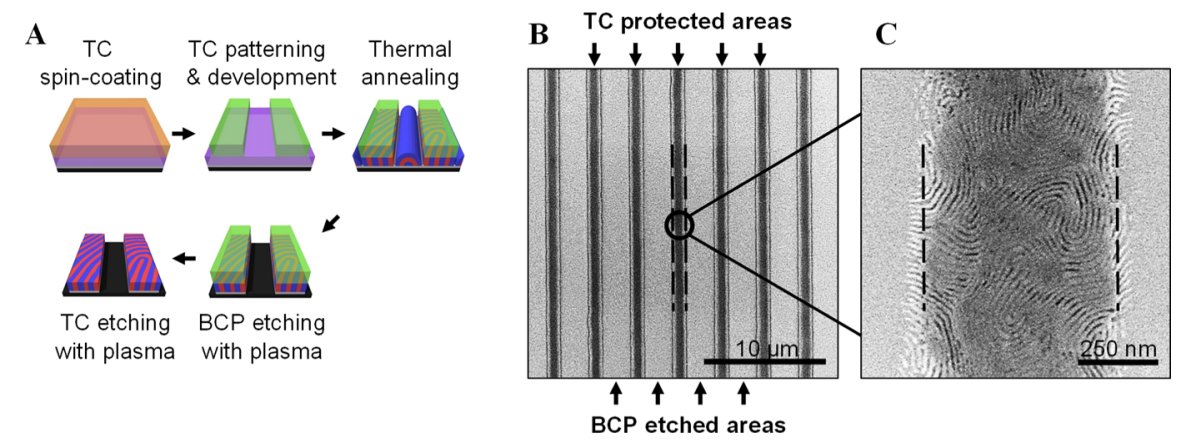

Figure 6. (A) Integration scheme allowing the definition of selected perpendicular areas in an L-DBS layer via patterning of the $90 \mathrm{~nm}$ thick TC protective layer using $365 \mathrm{~nm}$ lithography. (B) Low-magnification SEM top-view image of the BCP stripes protected by a FGH-37 TC layer obtained after etching the BCP dewetted areas. (C) SEM top-view image of the out-of-plane lamellar L-DBS18 structure revealed after etching of the protecting TC layer. The dashed lines are a guide to the eye to highlight the borders between BCP-perpendicular areas and featureless areas.

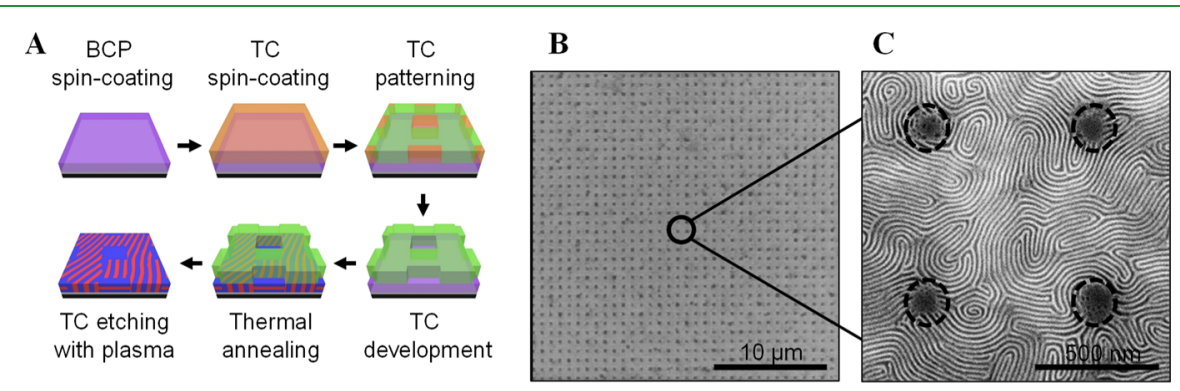

Figure 7. (A) Integration scheme illustrating the principle of TC-patterning with the confinement of capillary waves. (B) Low-magnification SEM top-view image of the FGH-37 N-TC patterned with electron-beam lithography according to (A) on L-DBS18, thermally annealed, and removal of the N-TC with selective plasma etching. The dark areas are the areas unexposed to the electron beam. (C) High-magnification SEM top-view image of (B). The dashed lines are a guide to the eye to highlight the borders between BCP-perpendicular areas and featureless areas.

the BCP layer but also readily inhibit the appearance of other mechanical instabilities, which are potential issues for further integration in microelectronics.

The thickness nonuniformity of sensitive resists in lithography directly affects the performances of IC devices from chip-to-chip, as dimensional variations become more critical with the nanometric scaling down of the features. ${ }^{49}$ The BCP film dewetting, leading to highly nonuniform coatings, is therefore detrimental in this regard. ${ }^{22}$ Its suppression is a sine qua non condition for a particular BCP system to fit into the stringent uniformity requirements imposed to thin-film resists for lithography-dedicated applications. ${ }^{50}$ As the designed TC materials demonstrated a tight control on the dewetting of the BCP layer, we further exploited their unique characteristics to implement specific lithographic capabilities as an essential point of this technology.

2.3. Novel Integration Schemes. A core feature of our TCs is their intrinsic ability to be patterned through standard lithographic processes. Indeed, the epoxy-methacrylate FGH platform is transparent at wavelengths down to $193 \mathrm{~nm}^{51}$ for meaningful resist thicknesses (i.e., from 50 to $100 \mathrm{~nm}$ ). ${ }^{52}$ It is thus easily scalable onto substrates with sizes of interest for the semiconductor industry (Figure 5A) and compatible with the most advanced patterning technologies, including the recently introduced extreme UV lithography ${ }^{53}$ for which typical resist thicknesses are in the range of $20-30 \mathrm{~nm} .^{54}$ This unique patterning property enables attractive DSA integration schemes via the definition of dedicated perpendicular and parallel areas into the BCP layer, as illustrated in Figure 5B. More precisely, the final selection of areas of interest inside the BCP layer is based either on direct lithographic approaches or on tunable TC affinities, as demonstrated in the following detailed examples.

Figure 6A illustrates a lithographic scheme where a $90 \mathrm{~nm}$ thick FGH-37 neutral TC (N-TC) layer deposited on top of the BCP film is first patterned using UV-365 nm photolithography and acts as a plasma-etch protecting layer. Following the development of the unexposed areas in ethanol and the promotion of the BCP self-assembly by a thermal annealing step at $240{ }^{\circ} \mathrm{C}$ for $5 \mathrm{~min}$, the dewetted BCP material located between the patterned areas (see Figure S6A) is removed by a nonselective plasma (this treatment also removes the upper part of the N-TC) (Figure 6B). A final $\mathrm{O}_{2}$-based plasma eventually ashes the remaining N-TC layer, leaving perpendicularly oriented $\mathrm{BCP}$ domains exclusively on selected areas (Figure 6C).

Figure 7A displays a related lithographic integration scheme taking further advantage of capillary force lithography principles. $^{55}$ Here, the FGH-37 N-TC is patterned with electron beam lithography in order to define features with a confining distance between two adjacent patterns below the critical wavelength of the capillary wave growth. As a result, the dewetting kinetics of the BCP film is suppressed during the thermal annealing step due to the confinement (see Figure $\mathrm{S} 6 \mathrm{~B}, \mathrm{C})$, and the corresponding noncovered $\mathrm{BCP}$ areas are therefore unable to dewet (Figure $7 \mathrm{~B}$ )..$^{56-58}$ This process concomitantly yields a change in the orientation of the BCP structure for uncovered areas, as the intrinsic surface energy difference between the blocks dictates the formation of an inplane structure ${ }^{59}$ as observed in Figure $7 \mathrm{C}$.

We further implemented a "TC affinity" approach relying on the use of TCs with different interfacial tensions: a N-TC and an affine-TC (A-TC) (affine being defined as preferential for 
A

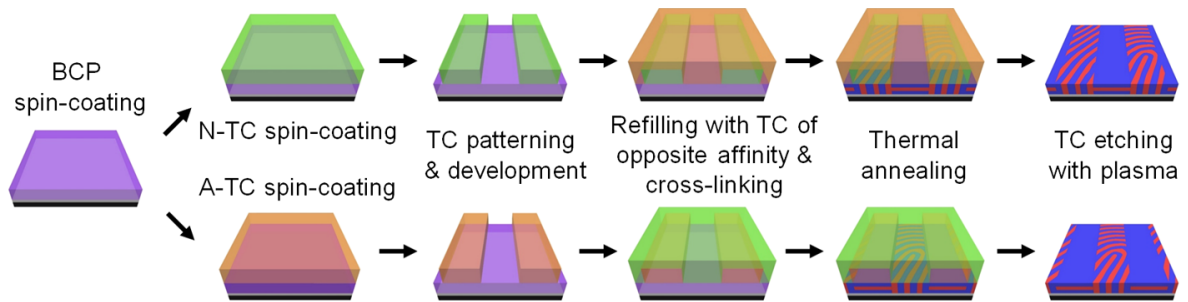

B N-TC patterning

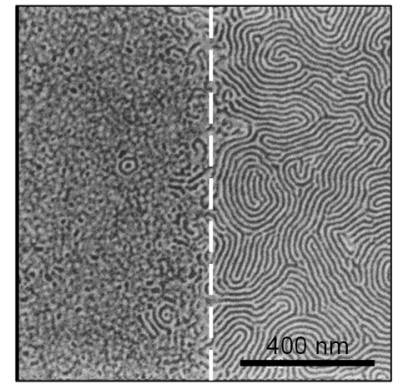

Affine spaces Neutral lines
C A-TC patterning

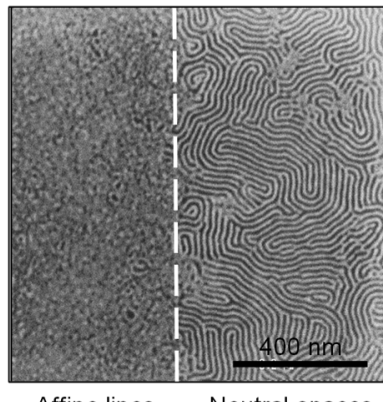

D

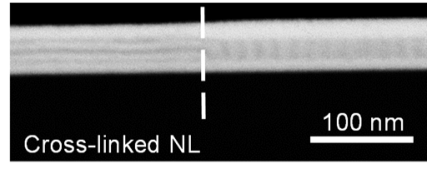

E

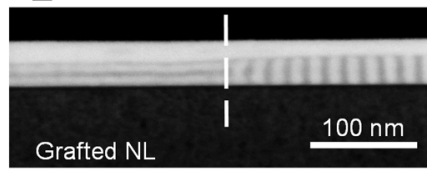

Figure 8. (A) Integration scheme for the "TC affinity" approach allowing the selection of perpendicular areas in a L-DBS layer via patterning of a $50 \mathrm{~nm}$ TC layer using $365 \mathrm{~nm}$ lithography. (B,C) SEM top-view images of in-plane or out-of-plane lamellar L-DBS18 BCP structures demarcated by the "TC affinity" approach after selective plasma etching of the A-TC (FGH-73 or FGH-20) and N-TC (FGH-47). (D,E) FIB-STEM crosssectional images of the interface between in-plane and out-of-plane lamellar structures observed in L-DBS18 layers following the "TC affinity" approach integrated on both (D) cross-linked FGH (FGH-47 and FGH-20 as N-TC and A-TC, respectively) or (E) grafted neutral layers (FGH47 and FGH-73 as N-TC and A-TC, respectively). The dashed lines in (B-E) are a guide to the eye to highlight the demarcation between the inplane and out-of-plane orientations of the BCP structure.

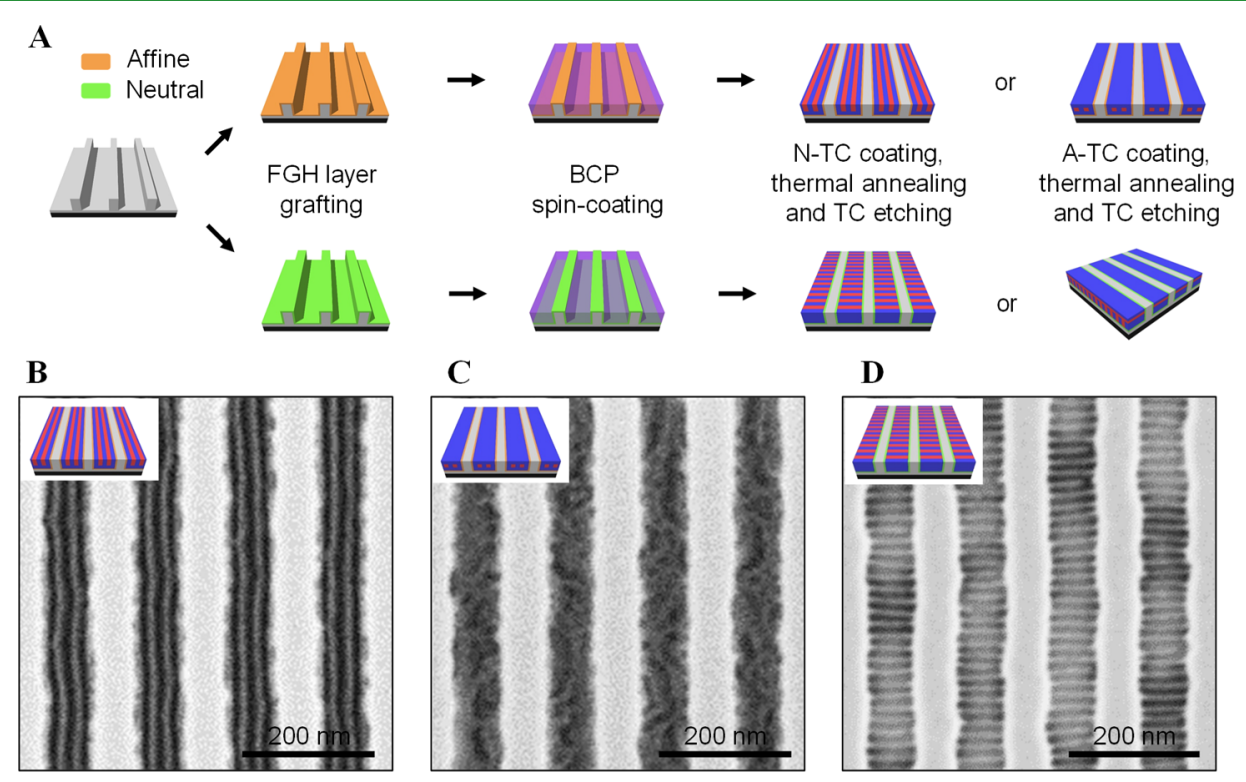

Figure 9. (A) Integration schemes illustrating the use of various interfacial configurations in combination with topographical template for the DSA of L-DBS18 BCP. (A-D) SEM top-view images of the resulting experimental nanostructures: (B) FGH-37 N-TC combined with DB-affine gratings leading to a "line \& space"-type pattern, (C) FGH-20 A-TC combined either neutral or affine gratings leading to a parallel orientation of lamellae, (D) FGH-37 N-TC combined with neutral gratings leading to a "ladder"-type pattern.

one of the blocks), readily depending on the FGH terpolymer composition. As shown in Figure 8A, a second cross-linkable TC material is used to refill the empty spaces created in the previously patterned TC, thus avoiding the dewetting of the BCP layer during thermal annealing, as described earlier in Figure 6A. Either N-TC or A-TC can be used as both patterned and refilling materials, thus enabling a BCP pattern transfer through the positive or negative spaces. Both pathways are exemplified in Figure 8B,C using UV-365 nm lithography as a patterning technique. An excellent control over the quality of nondewetted perpendicular and parallel areas with a sharp interface is experimentally observed following these integration schemes. As evidenced by the FIB-STEM cross-sectional image reported in Figure 8D,E, the interface between the two distinct orientations is also well-defined throughout the $\mathrm{BCP}$ film thickness.

The different TC-patterning schemes can be further applied to DSA methods guiding BCP structures, as illustrated in 
A

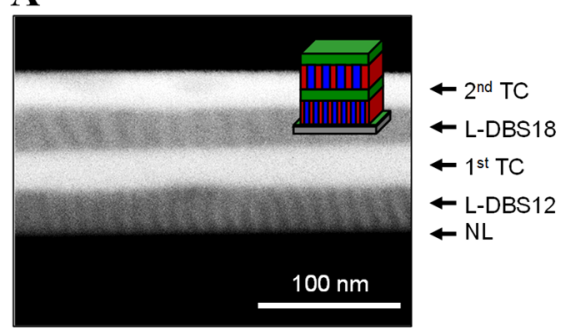

C

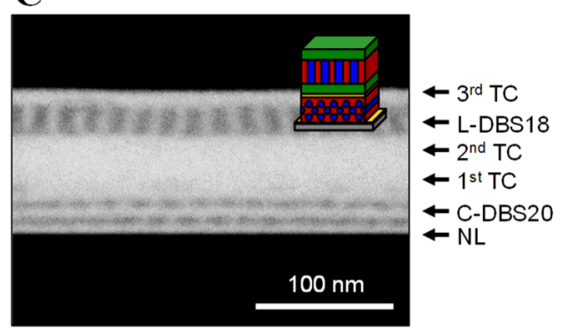

B

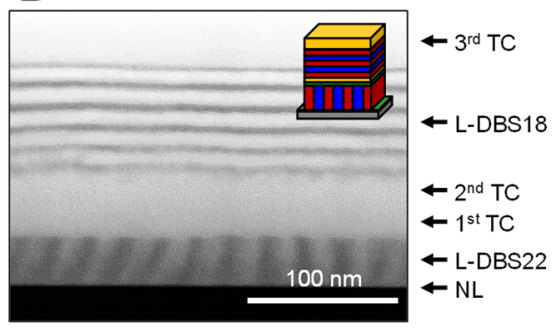

D

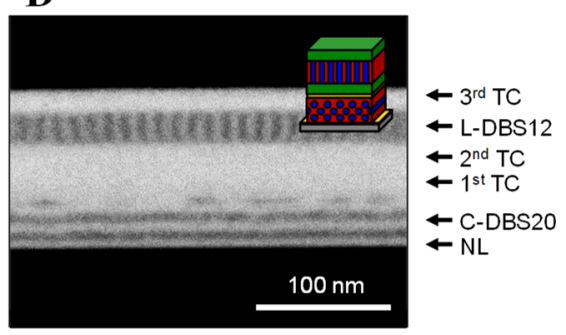

Figure 10. Three-dimensional structures obtained by iterative self-assembly of nanostructured DBS layers, enabled by interface manipulations via the introduction of FGH interlayers. FIB-STEM images of (A) NL/L-DBS12/TC\#1 (FGH-37)/L-DBS18/TC\#2 (FGH-37), (B) NL/L-DBS22/ TC\#1 (FGH-37)/TC\#2 (FGH-47)/L-DBS18/TC\#3 (FGH-20), (C) NL/C-DBS20/TC\#1 (FGH-24)/TC\#2 (FGH-47)/L-DBS18/TC\#3 (FGH37), and (D) NL/C-DBS20/TC\#1 (FGH-24)/TC\#2 (FGH-47)/L-DBS12/TC\#3 (FGH-24). The multilayers were thermally annealed at $260{ }^{\circ} \mathrm{C}$ during $5 \mathrm{~min}$ after the fabrication of the multilayered structures. Slightly tilted features are mainly due to the abrasive conditions used during FIBSTEM sample preparation. The shaded areas observed (in (A) or (D) for instance) are due to the polycrystalline nature of each independent BCP film, allowing random orientation of BCP grains (in (D) the C-DBS20 layer is observed along the long axis of the DB cylinders, while the cut is orthogonal to the lamellae for the L-DBS12).

Figure 9A using graphoepitaxy. In these integration schemes, the topographical substrate is modified through the grafting of underlayers with varying affinities to obtain either neutral or slightly affine sidewalls. The addition of a $\mathrm{N}$ - or A- TC completes the global integration scheme, leading to various combinations of directional fields for the control of the BCP, thus showcasing an example of a pathway toward future selection of areas of interest with such an approach. For the slightly affine template associated with a N-TC (top panel of Figure 9A), the DB phase of the BCP is attracted to the walls leading to a line and space pattern for satisfied commensurability conditions between the template and the BCP period, as shown in Figure 9B. For A-TC associated to either affine or neutral gratings, the directional field induced by the sidewalls is not strong enough to counter-balance the stronger affine top surface field arising from the A-TC. Consequently, the orientation of the BCP structure switches to a parallel configuration, as attested by the featureless SEM image (Figure 9C). ${ }^{60}$ In the case of neutral gratings combined with a N-TC (bottom panel of Figure 9A), the nanostructured LDBS18 layer thermally annealed at $240{ }^{\circ} \mathrm{C}$ results in the typical ladder pattern as neutral boundary conditions are insured at all interfaces (see Figure 9D). ${ }^{61}$ It is noteworthy that gratings of different affinities can also be generated with a thick crosslinked FGH layer obtained through a hot-embossing process by nanoimprint lithography and subsequently used as guiding templates for the BCP, as demonstrated in Figure S7. These results illustrate how the unique lithographic properties and patterning strategies of the FGH platform can be implemented with DSA-dedicated guiding templates to select perpendicular BCP features and thus simplify existing integration schemes (e.g., the "cut" step to obtain FinFETs and the patterning of contact layers for future CFETs or nanosheet-based devices). ${ }^{25,26}$
The aforementioned key-properties of the FGH materials were also advantageously exploited to enable the formation of three-dimensional stacks of BCP thin films. Such multilayered structures are foreseen to be valuable in cutting-edge technologies from photonics to microelectronics ${ }^{62,63}$ by expanding the breadth of nontrivial structures obtained by BCP self-assembly. ${ }^{64,65}$ Our approach relies on the direct deposition of a subsequent BCP layer on top of an immobilized TC, providing a straightforward and swift integration pathway with regards to existing iterative selfassembly methods. ${ }^{6-68}$ In addition, the unique lithographic properties of the FGH materials render them extremely appealing for the multilayering of BCP nanofeatures with the possibility of a spatially resolved control of the BCP structure orientation for each layer, while insuring the mechanical stability of the stacked layers and keeping the self-assembly capabilities of each BCP layer along the whole manufacturing process. Such characteristics are of significant relevance to achieve multilayered BCP stacks, as most of previously reported iterative methods either necessitate the deposition of inorganic species to avoid the resolubilization of each BCP layer ${ }^{68}$ or the use of cross-linkable BCPs architectures. ${ }^{69}$ Figure 10 illustrates the versatility of this stacking approach for a variety of combinations between BCP systems with different morphologies and periodicities. Figure 10A shows a doublelayer lamellar structure with an out-of-plane orientation of the BCP features with different periodicities. Additionally, the choice of the intermediate stack of cross-linked FGH layers with different affinities allows an independent control of the orientation of each BCP structure. As shown in Figure 10B, such a process generates a sequence of out-of-plane and inplane lamellar structures through the tuning of the FGH composition to manipulate the interfacial affinity. We further implemented this methodology to BCP systems with different 


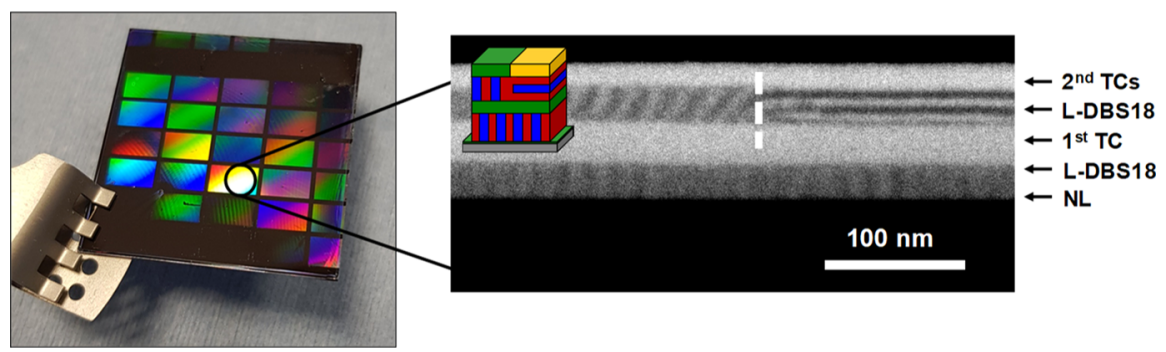

Figure 11. FIB-STEM images of three-dimensional structures obtained by iterative self-assembly of nanostructured DBS layers with a patterned upper interface. The N-TC component of the second TC layer was patterned through UV $365 \mathrm{~nm}$ exposure according to Figure 8A,D. The materials NL/L-DBS18/TC\#1 (FGH-37)/L-DBS18/TCs\#2 (FGH-37 and FGH-20 as N-TC and A-TC, respectively) are used accordingly. The left panel illustrates an optical image of the patterned sample used for the FIB-STEM characterization. The multilayers were thermally annealed at $260{ }^{\circ} \mathrm{C}$ during $5 \mathrm{~min}$ after the fabrication of the multilayered structures.

morphologies and multilayers based on the stacking of in-plane cylindrical, and out-of-plane lamellar structures were produced, as illustrated in Figure 10C,D. The specific patterning properties of the $\mathrm{FGH}$ materials and their unique integration schemes were combined to a stacking experiment, as illustrated in the Figure 11, to obtain perpendicular orientation of features inside selected areas of a particular BCP layer in the stack. It further contributes to demonstrate the versatility of our TC concept and opens up highly interesting perspectives for their use into 3D-stacking BCP opportunities.

\section{CONCLUSIONS}

We demonstrate here the implementation of new TC materials, soluble in benign alcoholic solvents, and able to be readily spin-coated on top of the BCP with a high uniformity at the wafer scale. Their use in conjunction with a Si-containing high- $\chi$ BCP system, promoting the formation of $\sim 6 \mathrm{~nm}$ resolved domains, enables the transfer of high aspect-ratio functional features into the substrate. The cross-linking ability inherent to the TC design produces a stiff layer on top of the viscoelastic $\mathrm{BCP}$ film and alleviates the critical issue of $\mathrm{BCP}$ dewetting while enabling the perpendicular orientation of the self-assembled features, essential for nanomanufacturing processes based on DSA. Their hybrid nature as patternable interfacial layers, at the cross-point between DSA-dedicated materials and conventional lithographic resists, leverages attractive integration schemes with spatially controlled orientation of BCP features. As key demonstrations, we exemplify several dedicated integration schemes, together with the use of this TC platform for the generation of nanostructured three-dimensional BCP multilayers. We anticipate that this holistic approach based on multifunctional TCs will greatly facilitate the implementation of DSA based on BCP self-assembly into functional devices and open up new horizons in several active scientific and technological fields (beyond-Complementary Metal-Oxide-Semiconductor (CMOS) devices, metamaterials, and biosensors) incorporating DSA-based nanostructures.

\section{EXPERIMENTAL SECTION}

4.1. Substrates. Unless otherwise mentioned, experiments were performed on $27 \times 27 \mathrm{~mm}^{2}$ [1,0,0]-oriented silicon samples cleaved from $200 \mathrm{~mm}$ diameter monitor wafers and used without further preparation.

4.2. Synthesis of FGH Terpolymers. Synthesis of the FGH terpolymers was performed by classical radical polymerization in solution. All the monomers, solvent, initiator, and terminating agent were used directly as received. As an example, in a $1 \mathrm{~L}$ vessel equipped with a mechanical stirrer, after one vacuum/nitrogen cycle, were successively introduced at room temperature, under nitrogen and stirring at $100 \mathrm{rpm}, 408.6 \mathrm{~g}$ of propylene glycol methyl ether (PGME or 1-methoxy-2-propanol from Technic), $35.0 \mathrm{~g}$ of glycidyl methacrylate (Daja Labs), $84.0 \mathrm{~g}$ of 2-hydroxyl ethyl methacrylate (Aldrich), and $21.0 \mathrm{~g}$ of 2,2,2-trifluoro ethyl methacrylate (Aldrich). Three additional vacuum/nitrogen cycles were applied to the reactor and then under nitrogen, heating the solution at $100{ }^{\circ} \mathrm{C}$, under stirring at $100 \mathrm{rpm}, 10.2 \mathrm{~g}$ of dodecanethiol (Aldrich, pu. 98\%) and $1.4 \mathrm{~g}$ of 2,2'-azobis (2-methylpropionitrile) (AIBN-Arkema) in solution in $70.0 \mathrm{~g}$ of PGME were added. After $3 \mathrm{~h}$, an additional solution of $1.4 \mathrm{~g}$ of AIBN in $70.0 \mathrm{~g}$ of PGME was injected and the polymerization was completed in $3 \mathrm{~h}$. The polymer is recovered from the solution after drying under vacuum in an oven $12 \mathrm{~h}$ at $60{ }^{\circ} \mathrm{C}$.

The macromolecular characterization of the FGH terpolymers was performed by using a combination of size-exclusion chromatography using poly(styrene) standards for the determination of the molecular weight and ${ }^{1} \mathrm{H}$ NMR spectroscopy for the terpolymer composition (Table 1).

4.3. Synthesis of DBS Block Copolymers. 1,1-Dimethyl silacyclobutane (DB) (Sigma-Aldrich, 95\%) and styrene (S) (Across Organic, 99.9\%) were distilled twice over $\mathrm{CaH}_{2}$ and di- $n$ butylmagnesium, respectively. Tetrahydrofuran (THF) and heptane were first distilled over $\mathrm{CaH}_{2}$ and then over sodium/benzophenone and living poly(styrene), respectively. The DBS BCPs were synthesized via anionic polymerization by sequential addition of the $\mathrm{DB}$ and $\mathrm{S}$ monomers in a THF/heptane mixture (50/50 in volume) at $-50{ }^{\circ} \mathrm{C}$ using sec-BuLi as an initiator. The macromolecular characterization of the BCPs was performed by using a combination of size-exclusion chromatography using the universal calibration technique for the determination of the molecular weight and ${ }^{1} \mathrm{H}$ NMR spectroscopy for the block copolymer composition (Table S1).

4.4. Preparation of Interfacial Layers. The grafted FGH underlayers were produced by spin coating (700 rpm) a 2 wt \% terpolymer solution in methyl isobutyl ketone (MIBK), followed by a thermal annealing step at $200{ }^{\circ} \mathrm{C}$ for 75 s. Rinsing with PGMEA results in a grafted FGH layer with $5 \mathrm{~nm}$ thickness. The cross-linked FGH underlayers were obtained by spin coating ( $2 \mathrm{krpm})$ a 2 wt $\%$ terpolymer solution in MIBK loaded with a $0.2 \mathrm{wt} \%$ of ITC or PAG (ammonium trifluoromethanesulfonate as ITC (4-phenylthiophenyl)diphenylsulfonium trifluoromethanesulfonate as PAG). The thermal activation of the cross-linking was obtained by an annealing step at $240{ }^{\circ} \mathrm{C}$ for $2 \mathrm{~min}$, unless otherwise stated. The photoactivation of the cross-linking was performed using a $365 \mathrm{~nm}$ wavelength with a dose of $100 \mathrm{~mJ} \cdot \mathrm{cm}^{-2}$, followed by a first postexposure bake at $90{ }^{\circ} \mathrm{C}$ during $3 \mathrm{~min}$ and a thermal annealing step at $240{ }^{\circ} \mathrm{C}$ for $2 \mathrm{~min}$.

The cross-linked FGH layers used as top coats were obtained from a 2 wt \% terpolymer solution in a mixture of PGME and ethanol (1/ 1) with a 0.2 wt $\%$ of ITC or PAG spin-coated (2 krpm) on top of the BCP film and annealed at $60{ }^{\circ} \mathrm{C}$ for $1 \mathrm{~min}$. The resulting TC layer with approximatively $50 \mathrm{~nm}$ thickness is then cured at $90^{\circ} \mathrm{C}$ for $3 \mathrm{~min}$ (thermally-activated TC) or exposed to $365 \mathrm{~nm}$ wavelength with a dose of $100 \mathrm{~mJ} \cdot \mathrm{cm}^{-2}$ and a postexposure bake at $90{ }^{\circ} \mathrm{C}$ for $3 \mathrm{~min}$ 
(photo-activated TC) before the promotion of the BCP self-assembly by thermal annealing.

4.5. Preparation of BCP Films. DBS solutions ( 0.9 wt \%) in MIBK were spin-coated (2 krpm) on immobilized NLs followed by a soft bake at $60{ }^{\circ} \mathrm{C}$ for $1 \mathrm{~min}$ to produce $\mathrm{BCP}$ layers with approximatively $30 \mathrm{~nm}$ thickness.

4.6. Multilayered 3D Structures. As an example, a double stack of lamellar L-DBS12 on cylindrical C-DBS20 (Figure 6d) on Si was prepared as follows: end-functionalized polystyrene $(\mathrm{OH}$-terminated, $M_{\mathrm{w}}=11 \mathrm{~kg} \cdot \mathrm{mol}^{-1}$ ) dissolved at $3.6 \mathrm{wt} \%$ in MIBK was spin-coated at $2000 \mathrm{rpm}$ on monitor $\mathrm{Si}$ and thermally grafted at $200^{\circ} \mathrm{C}$ for $75 \mathrm{~s}$. The sample was rinsed in PGMEA to remove excess PS and dried under nitrogen flow. The first BCP layer of C-DBS20 at $1 \mathrm{wt} \%$ in MIBK was spin-coated at $1000 \mathrm{rpm}$ and the solvent evaporated at $60{ }^{\circ} \mathrm{C}$ for 1 min. A solution of FGH-24 with PAG at 2 and 0.2 wt \% in ethanol, respectively, was diluted with PGME to reach ethanol/PGME $=95: 5$, spin-coated at $2000 \mathrm{rpm}$, the solvent was evaporated at $60{ }^{\circ} \mathrm{C}$ for 1 $\mathrm{min}$, the sample placed under UV irradiation for $180 \mathrm{~s}$ to activate the PAG initiator, and the cross-linking reaction was continued at $90{ }^{\circ} \mathrm{C}$ for $3 \mathrm{~min}$, followed by $240^{\circ} \mathrm{C}$ for $2 \mathrm{~min}$. This sequence of steps was repeated with a solution of FGH-47 with PAG (ethanol/PGME 9:1, all other parameters equal) to produce a neutral underlayer for the second BCP layer. L-DBS12 at 1 wt \% in MIBK was spin-coated at $2000 \mathrm{rpm}$ and the solvent evaporated at $60^{\circ} \mathrm{C}$ for $1 \mathrm{~min}$. The solution of FGH-24 with PAG deposited and cross-linked as described above was used as the final top coat, with a cure at $260{ }^{\circ} \mathrm{C}$ for $5 \mathrm{~min}$ to allow complete self-assembly of both BCPs.

4.7. Thermal and UV Annealing. Thermal annealing to promote self-organization of the BCP or cross-linking of interfacial materials was carried out on standard hot plates in a cleanroom environment $\left(23{ }^{\circ} \mathrm{C}, 45 \% \mathrm{RH}\right)$. UV irradiation was performed with a PanacolEleco UVP280/2, nonfiltered, fibered, mercury arc lamp with a fluence of $1.6 \mathrm{~mW} / \mathrm{cm}^{2}$ at a wavelength of $365 \mathrm{~nm}$. Unless otherwise stated, the exposure time was $3 \mathrm{~min}$.

4.8. Plasma Etching. Etching experiments were performed in a $300 \mathrm{~mm}$ AdvantEdge MESA ICP etch tool from Applied Materials with chamber walls made of yttrium oxide. Samples were patched on $300 \mathrm{~mm} \mathrm{Si}$ wafers. To allow good process reproducibility, the chamber was first cleaned using a three-step plasma: $\mathrm{NF}_{3} / \mathrm{Cl}_{2}$ for $45 \mathrm{~s}$, $\mathrm{Cl}_{2} / \mathrm{O}_{2} / \mathrm{Ar}$ for $45 \mathrm{~s}$, and finally $\mathrm{O}_{2}$ for $30 \mathrm{~s}$.

The transfer of the lamellar BCP features into the silicon substrate was performed using a multistep process: (i) the chamber was conditioned using a $\mathrm{H}_{2} / \mathrm{N}_{2}$ plasma for 5 min on a dummy wafer; (ii) a $\mathrm{H}_{2} / \mathrm{N}_{2}$ plasma was used for $30 \mathrm{~s}$ in order to remove the cross-linked TC layer; (iii) the selective removal of the PS domains was carried out with a $\mathrm{N}_{2} / \mathrm{O}_{2}$ plasma for $35 \mathrm{~s}$ in a carbon-coated chamber (achieved beforehand using a $\mathrm{CH}_{4}$-based plasma on a dummy wafer); (iv) the oxidized $\mathrm{DB}$ features were finally transferred into the $\mathrm{Si}$ substrate using a $\mathrm{Cl}_{2}$ breakthrough and a dedicated pulsed $\mathrm{HBr} / \mathrm{O}_{2}$ plasma.

Partial etching of the BCP films were required in specific experiments: the nonselective etching of the $\mathrm{BCP}$ layer with respect to the TC was achieved using a $\mathrm{O}_{2} / \mathrm{CF}_{4}(50 / 50)$ plasma, while the selective etching of the TC with respect to the $\mathrm{BCP}$ was achieved using a $\mathrm{Ar} / \mathrm{O}_{2}$ plasma.

4.9. Small-Angle X-ray Scattering. SAXS experiments were performed on the D2AM French-CRG Beamline at the European Synchrotron Radiation Facility (ESRF) in France. A XPAD 2D pixel detector was positioned at a $2400 \mathrm{~mm}$ sample-to-detector distance for recording the $2 \mathrm{D}$ scattering images. The energy of the X-ray beam was $11 \mathrm{keV}$. The $2 \mathrm{D}$ images were radially averaged around the center of the primary beam to obtain the isotropic SAXS intensity profiles. The scattering pattern from a specimen of silver behenate was used for the calibration of the wave vector scale of the scattering curves. Finally, the data were normalized to the intensity of the incident beam to correct for primary beam intensity fluctuations.

4.10. Grazing Incidence Small-Angle X-ray Scattering. GISAXS experiments were performed on the ALBA Synchrotron in Spain using an X-ray beam energy of $8 \mathrm{keV}$ and a sample-to-detector distance of $3048 \mathrm{~mm}$. The incidence angle was set at $0.15^{\circ}$, which is close to the critical angle of the $\mathrm{BCP}$ film. $2 \mathrm{D}$ scattering patterns were collected using a PILATUS $1 \mathrm{M}$ Dectris detector and were reduced using the GIXSGUI package, ${ }^{70}$ where $q_{y}=2 \pi / \lambda\left[\sin \left(2 \theta_{f}\right) \cos \left(\alpha_{f}\right)\right]$ and $q_{z}=2 \pi / \lambda\left[\sin \left(\alpha_{f}\right)+\sin \left(\alpha_{i}\right)\right]$ are the modulus of the scattering vectors in the direction parallel and perpendicular to the substrate plane and $\alpha_{i}, 2 \theta_{f}$, and $\alpha_{f}$ are the incident and scattering angles in the horizontal and vertical directions, respectively.

4.11. Dewetting Experiments. The different multilayered configurations used for the dewetting experiments were prepared according to the aforementioned processes. The multilayers were then thermally-annealed at temperatures from 90 to $260{ }^{\circ} \mathrm{C}$ during $5 \mathrm{~min}$ in order to mimic the promotion of the BCP self-assembly. The TC layers were subsequently etched with an ICP plasma based on $\mathrm{O}_{2}$ or $\mathrm{Ar} / \mathrm{O}_{2}$ chemistries in order to reveal the BCP layer. The samples were then observed with a CD-SEM instrument at a magnification of $\times 5000$ to give $27 \mu \mathrm{m} \times 27 \mu \mathrm{m}$ field-area (or magnification $\times 10000$ with field area of $13.5 \mu \mathrm{m} \times 13.5 \mu \mathrm{m}$ ), and around 10 images were randomly recorded for each sample. The pictures were then postprocessed using Image analysis software from the National Institutes of Health (NIH) (https://imagej.nih.gov/ij/) in order to quantify the proportion of nondewetted areas.

4.12. SEM and STEM. Top-view SEM images were acquired on a Hitachi CD-SEM H-9300 microscope. Typical parameters for the observations were $0.5 \mathrm{kV}$ electron acceleration voltage with $6 \mu \mathrm{A}$ current. Cross-section SEM images were acquired on a Hitachi S5000 microscope at $30 \mathrm{kV}$ electron acceleration voltage with $10 \mu \mathrm{A}$ current. Sample preparations for STEM were carried out using a Helios 450S-FEI dual-beam microscope. A $0.5 \mu \mathrm{m}$ thick Pt protective layer was deposited on top of the sample inside the equipment using a high-energy ion beam. A thin carbon layer was deposited beforehand on top of the Si samples to accentuate the contrast. A $30 \mu \mathrm{m} \times 2 \mu \mathrm{m}$ specimen is then etched away from the surface and thinned to approximately $100 \mathrm{~nm}$ width. A transmission image of the crosssection is finally recorded in scanning mode at $29 \mathrm{kV}$ and $50 \mathrm{pA}$ on the same equipment.

4.13. AFM Nanoindentation. AFM (Dimension FastScan, Bruker) was used in Nanoindention mode (Quantitative Nanomechanical Property Mapping) to obtain the Young's modulus of the BCP/TC stacks. Tapping probes (RTESPA-300, Bruker) with a spring constant of $40 \mathrm{~N} \cdot \mathrm{m}^{-1}$ were used.

4.14. Surface Energy Measurements. The contact angles of water, ethylene glycol, and di-iodomethane (10 measurements for each liquid) were measured using a drop shape analyzer (DSA100, $\mathrm{KRÜSS} \mathrm{GmbH})$ in a cleanroom environment $\left(23{ }^{\circ} \mathrm{C}, 45 \% \mathrm{RH}\right)$. Surface energy values were determined via the work method, directly calculated by the Advance software provided by KRÜSS GmbH.

4.15. Ellipsometry. Uniformity of the spreading of the various polymeric layers was probed using spectroscopic ellipsometry in reflection mode with a phase modulated spectroscopic ellipsometer (UVISEL, from Horiba Scientific) on the spectral range 200-800 nm.

4.16. Nanoimprint, UV, and Electron-Beam Lithography. Silicon molds used for thermal nanoimprint experiments were prepared by standard dry $193 \mathrm{~nm}$ lithography and plasma etching on $300 \mathrm{~mm}$ wafers. An individual chip of approximately $1 \mathrm{~cm}^{2}$ was then treated with an antisticking layer (Optool DSX from Daikin, Japan) and used repeatedly as a mold to form gratings in a FGH layer with approximatively $45 \mathrm{~nm}$ thickness ( 50 bars for $5 \mathrm{~min}$ at $50{ }^{\circ} \mathrm{C}$ followed by 50 bars for $2 \mathrm{~min}$ at $240{ }^{\circ} \mathrm{C}$ ).

UV lithography on $4 \mathrm{in}$. wafers and $27 \times 27 \mathrm{~mm}^{2}$ silicon chips was carried out with a Karl Süss MJB4 equipment equipped with a mercury arc lamp having a power of $20 \mathrm{~mW} / \mathrm{cm}^{2}$ at a wavelength of $365 \mathrm{~nm}$ using a typical exposure time of $50 \mathrm{~s}$.

Electron-beam lithography was performed on a JEOL6300FS equipment on $20 \times 20 \mathrm{~mm}^{2}$ samples at an acceleration voltage of 100 $\mathrm{kV}$, with a current of $500 \mathrm{pA}$ and a dose of $80 \mu \mathrm{C} / \mathrm{cm}^{2}$. 
Detailed analysis of uniformity mapping of FGH material, detailed self-assembly cartographies related to Figure 1, chemical and morphological structure of the BCP used, Young's modulus of a BCP/TC stack, SEM images of a nanostructured BCP thin films after rapid thermal annealing, dewetting experiments with electronbeam patterned TC, graphoepitaxy with nanoimprintfabricated FGH-material templates, macromolecular characteristics of the DBS copolymers, surface energy of the FGH materials, and discussion on dewetting of nanostructured BCP films on "neutral" layers (PDF)

\section{AUTHOR INFORMATION}

\section{Corresponding Authors}

Xavier Chevalier - ARKEMA FRANCE, GRL, BP34 64170

Lacq, France; $\odot$ orcid.org/0000-0001-7397-7849;

Email: xavier.chevalier@arkema.com

Guillaume Fleury - University of Bordeaux, CNRS, Bordeaux INP, LCPO, F-33600 Pessac, France;

Email: guillaume.fleury@u-bordeaux.fr

Marc Zelsmann - University of Grenoble Alpes, CNRS, CEA/ LETI Minatec, Grenoble INP, LTM, 38000 Grenoble,

France; Email: marc.zelsmann@cea.fr

\section{Authors}

Cindy Gomes Correia - University of Bordeaux, CNRS, Bordeaux INP, LCPO, F-33600 Pessac, France

Gwenaelle Pound-Lana - University of Grenoble Alpes, CNRS, CEA/LETI Minatec, Grenoble INP, LTM, 38000 Grenoble, France

Philippe Bézard - University of Grenoble Alpes, CNRS, CEA/ LETI Minatec, Grenoble INP, LTM, 38000 Grenoble, France

Matthieu Sérégé - University of Grenoble Alpes, CNRS, CEA/LETI Minatec, Grenoble INP, LTM, 38000 Grenoble, France

Camille Petit-Etienne - University of Grenoble Alpes, CNRS, CEA/LETI Minatec, Grenoble INP, LTM, 38000 Grenoble, France

Guillaume Gay - University of Grenoble Alpes, CNRS, CEA/ LETI Minatec, Grenoble INP, LTM, 38000 Grenoble, France

Gilles Cunge - University of Grenoble Alpes, CNRS, CEA/ LETI Minatec, Grenoble INP, LTM, 38000 Grenoble, France

Benjamin Cabannes-Boué - University of Bordeaux, CNRS, Bordeaux INP, LCPO, F-33600 Pessac, France

Célia Nicolet - ARKEMA FRANCE, GRL, BP34 64170 Lacq, France

Christophe Navarro - ARKEMA FRANCE, GRL, BP34 64170 Lacq, France

Ian Cayrefourcq - ARKEMA FRANCE, GRL, BP34 64170 Lacq, France

Marcus Müller - Georg-August Universität Göttingen, Institute for Theoretical Physics, 37077 Göttingen, Germany; (1) orcid.org/0000-0002-7472-973X

Georges Hadziioannou - University of Bordeaux, CNRS, Bordeaux INP, LCPO, F-33600 Pessac, France

Ilias Iliopoulos - Laboratoire PIMM, Arts et Métiers Institute of Technology, CNRS, Cnam, HESAM Université, 75013 Paris, France

\section{Author Contributions}

X.C. proposed the concept of rigid, cross-linkable and patternable TCs to circumvent dewetting of BCPs and conceived the FGH materials and the formulations. X.C., M.Z., G. F., I.I., and I.C designed the integration pathways. B.C.B., C.N., and C.N. synthesized the materials used in this study. X.C., C.G.C., G.P.-L., and M.Z. performed the self-assembly study. G.F. conducted the scattering and phys-icochemical characterizations. P.B., G.P.-L., M.S., C.P.-E., C.G., G.G., X.C., and M.Z. developed the etch-transfer and patterning methods. G.P.-L and M.S. performed the FIB- STEM characterizations. X.C., M.Z., and G.F. interpreted the experimental results. X.C. and G.F. wrote the manuscript with the contribution of M.Z., I.I., M.M., and G.H.. All authors have given approval to the final version of the manuscript.

\section{Notes}

The authors

declare the

following competing financial interest(s): X.C. and I. I. declare two different provisional patent applications (WO19102160A1 and WO19102158A1) submitted by Arkema to the INPI office, covering applicative aspects of this study.

\section{ACKNOWLEDGMENTS}

Prof. M. Geoghegan is gratefully acknowledged for helpful discussion. The authors thank S. Arnaud and G. Pecastaings for assistance with FIB-STEM and AFM nanoindentation experiments, respectively. The authors thank N. Demazy for the schematic illustrations of the patterning routes. This work was supported by the REX-7 project from Région Rhône Alpes and BPI France, by the French RENATECH network, and by the LabEx Minos ANR-10-LABX-55-01. C.G.C., G.H., and G.F. acknowledge financial support from the LCPO/Arkema Industrial Chair "HOMERIC" (ANR-13-CHIN-0002-01), the Equipex ELORPrintTec ANR-10-EQPX-28-01 with the help of the French state's Initiative d'Excellence IdEx ANR-10IDEX-003-02. I.I. acknowledges financial support from the Industrial Chair Arkema/CNRS-ENSAM-Cnam. The ESRF (D2AM beamline for the SAXS experiments) and the ALBA Synchrotron Light Source (BL11-NCD-SWEET beamline for the GISAXS experiments) are acknowledged for allocating beamtime.

\section{REFERENCES}

(1) Park, M.; Harrison, C.; Chaikin, P. M.; Register, R. A.; Adamson, D. H. Block Copolymer Lithography: Periodic Arrays of 1011 Holes in 1 Square Centimeter. Science 1997, 276, 1401-1404.

(2) Darling, S. B. Directing the Self-Assembly of Block Copolymers. Prog. Polym. Sci. 2007, 32, 1152-1204.

(3) Kim, H.-C.; Park, S.-M.; Hinsberg, W. D.; Division, I. R. Block Copolymer Based Nanostructures: Materials, Processes, and Applications to Electronics. Chem. Rev. 2010, 110, 146-177.

(4) Chai, J.; Wang, D.; Fan, X.; Buriak, J. M. Assembly of Aligned Linear Metallic Patterns on Silicon. Nat. Nanotechnol. 2007, 2, 500506.

(5) Robbins, S. W.; Beaucage, P. A.; Sai, H.; Tan, K. W.; Werner, J. G.; Sethna, J. P.; DiSalvo, F. J.; Gruner, S. M.; Van Dover, R. B.; Wiesner, U. Block Copolymer Self-Assembly-Directed Synthesis of Mesoporous Gyroidal Superconductors. Sci. Adv. 2016, 2, 1501119.

(6) Leibler, L. Theory of Microphase Separation in Block Copolymers. Macromolecules 1980, 13, 1602-1617. 
(7) Bates, F. S.; Fredrickson, G. H. Block Copolymer Thermodynamics: Theory and Experiment. Annu. Rev. Phys. Chem. 1990, 41, 525-557.

(8) Shin, K.; Leach, K. A.; Goldbach, J. T.; Kim, D. H.; Jho, J. Y.; Tuominen, M.; Hawker, C. J.; Russell, T. P. A Simple Route to Metal Nanodots and Nanoporous Metal Films. Nano Lett. 2002, 2, 933936.

(9) Bates, C. M.; Maher, M. J.; Janes, D. W.; Ellison, C. J.; Willson, C. G. Block Copolymer Lithography. Macromolecules 2014, 47, 2-12.

(10) Mansky, P.; Lui, Y.; Huang, E.; Russell, T. P.; Hawker, C. J. Controlling Polymer-Surface Interactions with Random Copolymer Brushes. Science 1997, 275, 1458-1460.

(11) Ouk Kim, S.; Solak, H. H.; Stoykovich, M. P.; Ferrier, N. J.; de Pablo, J. J.; Nealey, P. F. Epitaxial Self-Assembly of Block Copolymers on Lithographically Defined Nanopatterned Substrates. Nature 2003, 424, 411-414.

(12) Segalman, R. A.; Yokoyama, H.; Kramer, E. J. Graphoepitaxy of Spherical Domain Block Copolymer Films. Adv. Mater. 2001, 13, $1152-1155$

(13) Sinturel, C.; Bates, F. S.; Hillmyer, M. A. High $\chi$-LowNBlock Polymers: How Far Can We Go? ACS Macro Lett. 2015, 4, 10441050.

(14) Bates, C. M.; Seshimo, T.; Maher, M. J.; Durand, W. J.; Cushen, J. D.; Dean, L. M.; Blachut, G.; Ellison, C. J.; Willson, C. G. Polarity-Switching Top Coats Enable Orientation of Sub-10-Nm Block Copolymer Domains. Science 2012, 338, 775-779.

(15) Maher, M. J.; Bates, C. M.; Blachut, G.; Sirard, S.; Self, J. L.; Carlson, M. C.; Dean, L. M.; Cushen, J. D.; Durand, W. J.; Hayes, C. O.; Ellison, C. J.; Willson, C. G. Interfacial Design for Block Copolymer Thin Films. Chem. Mater. 2014, 26, 1471-1479.

(16) Suh, H. S.; Kim, D. H.; Moni, P.; Xiong, S.; Ocola, L. E.; Zaluzec, N. J.; Gleason, K. K.; Nealey, P. F. Sub-10-Nm Patterning via Directed Self-Assembly of Block Copolymer Films with a VapourPhase Deposited Topcoat. Nat. Nanotechnol. 2017, 12, 575-581.

(17) Lu, K.-Y.; Lo, T.-Y.; Georgopanos, P.; Avgeropoulos, A.; Shi, A.-C.; Ho, R.-M. Orienting Silicon-Containing Block Copolymer Films with Perpendicular Cylinders via Entropy and Surface Plasma Treatment. Macromolecules 2017, 50, 9403-9410.

(18) Oh, J.; Suh, H. S.; Ko, Y.; Nah, Y.; Lee, J.-C.; Yeom, B.; Char, K.; Ross, C. A.; Son, J. G. Universal Perpendicular Orientation of Block Copolymer Microdomains Using a Filtered Plasma. Nat. Commun. 2019, 10, 2912.

(19) Zhang, J.; Clark, M. B.; Wu, C.; Li, M.; Trefonas, P.; Hustad, P. D. Orientation Control in Thin Films of a High- $\chi$ Block Copolymer with a Surface Active Embedded Neutral Layer. Nano Lett. 2016, 16, $728-735$.

(20) Kim, D. H.; Kim, S. Y. Universal Interfacial Control through Polymeric Nanomosaic Coating for Block Copolymer Nanopatterning. ACS Nano 2020, 14, 7140-7151.

(21) Yoshida, H.; Suh, H. S.; Ramirez-Herunandez, A.; Lee, J. I.; Aida, K.; Wan, L.; Ishida, Y.; Tada, Y.; Ruiz, R.; de Pablo, J.; Nealey, P. F. Topcoat Approaches for Directed Self-Assembly of Strongly Segregating Block Copolymer Thin Films. J. Photopolym. Sci. Technol. 2013, 26, 55-58.

(22) Kim, T. H.; Hwang, J.; Hwang, W. S.; Huh, J.; Kim, H.-C.; Kim, S. H.; Hong, J. M.; Thomas, E. L.; Park, C. Hierarchical Ordering of Block Copolymer Nanostructures by Solvent Annealing Combined with Controlled Dewetting. Adv. Mater. 2008, 20, 522-527.

(23) Ferrarese Lupi, F.; Giammaria, T. J.; Miti, A.; Zuccheri, G.; Carignano, S.; Sparnacci, K.; Seguini, G.; De Leo, N.; Boarino, L.; Perego, M.; Laus, M. Hierarchical Order in Dewetted Block Copolymer Thin Films on Chemically Patterned Surfaces. ACS Nano 2018, 12, 7076-7085.

(24) Brassat, K.; Lindner, J. K. N. Nanoscale Block Copolymer SelfAssembly and Microscale Polymer Film Dewetting: Progress in Understanding the Role of Interfacial Energies in the Formation of Hierarchical Nanostructures. Adv. Mater. Interfaces 2020, 7, 1901565. (25) Tsai, H.; Pitera, J. W.; Miyazoe, H.; Bangsaruntip, S.; Engelmann, S. U.; Liu, C.-C.; Cheng, J. Y.; Bucchignano, J. J.;
Klaus, D. P.; Joseph, E. A.; Sanders, D. P.; Colburn, M. E.; Guillorn, M. A. Two-Dimensional Pattern Formation Using Graphoepitaxy of PS-b-PMMA Block Copolymers for Advanced FinFET Device and Circuit Fabrication. ACS Nano 2014, 8, 5227-5232.

(26) Liu, C.-C.; Franke, E.; Mignot, Y.; Xie, R.; Yeung, C. W.; Zhang, J.; Chi, C.; Zhang, C.; Farrell, R.; Lai, K.; Tsai, H.; Felix, N.; Corliss, D. Directed Self-Assembly of Block Copolymers for 7 Nanometre FinFET Technology and Beyond. Nat. Electron. 2018, 1, $562-569$.

(27) Huang, E.; Pruzinsky, S.; Russell, T. P.; Mays, J.; Hawker, C. J. Neutrality Conditions for Block Copolymer Systems on Random Copolymer Brush Surfaces. Macromolecules 1999, 32, 5299-5303.

(28) Girardot, C.; Böhme, S.; Archambault, S.; Salaün, M.; LatuRomain, E.; Cunge, G.; Joubert, O.; Zelsmann, M. Pulsed Transfer Etching of PS-PDMS Block Copolymers Self-Assembled in $193 \mathrm{~nm}$ Lithography Stacks. ACS Appl. Mater. Interfaces 2014, 6, 1627616282.

(29) Legrain, A.; Fleury, G.; Mumtaz, M.; Navarro, C.; Arias-Zapata, J.; Chevalier, X.; Cayrefourcq, I.; Zelsmann, M. Straightforward Integration Flow of a Silicon-Containing Block Copolymer for LineSpace Patterning. ACS Appl. Mater. Interfaces 2017, 9, 43043-43050.

(30) Krausch, G. Dewetting at the Interface between Two Immiscible Polymers. J. Phys. Condens. Matter 1997, 9, 7741-7752.

(31) Geoghegan, M.; Krausch, G. Wetting at Polymer Surfaces and Interfaces. Prog. Polym. Sci. 2003, 28, 261-302.

(32) Farrell, R. A.; Kehagias, N.; Shaw, M. T.; Reboud, V.; Zelsmann, M.; Holmes, J. D.; Sotomayor Torres, C. M.; Morris, M. A. Surface-Directed Dewetting of a Block Copolymer for Fabricating Highly Uniform Nanostructured Microdroplets and Concentric Nanorings. ACS Nano 2011, 5, 1073-1085.

(33) Xue, L.; Han, Y. Inhibition of Dewetting of Thin Polymer Films. Prog. Mater. Sci. 2012, 57, 947-979.

(34) Takahashi, H.; Laachi, N.; Delaney, K. T.; Hur, S.-M.; Weinheimer, C. J.; Shykind, D.; Fredrickson, G. H. Defectivity in Laterally Confined Lamella-Forming Diblock Copolymers: Thermodynamic and Kinetic Aspects. Macromolecules 2012, 45, 6253-6265.

(35) Li, W.; Müller, M. Defects in the Self-Assembly of Block Copolymers and Their Relevance for Directed Self-Assembly. Annu. Rev. Chem. Biomol. Eng. 2015, 6, 187-216.

(36) Li, W.; Müller, M. Thermodynamics and Kinetics of Defect Motion and Annihilation in the Self-Assembly of Lamellar Diblock Copolymers. Macromolecules 2016, 49, 6126-6138.

(37) Wyart, F. B.; Martin, P.; Redon, C. Liquid/Liquid Dewetting. Langmuir 1993, 9, 3682-3690.

(38) Jiang, J.; Jacobs, A. G.; Wenning, B.; Liedel, C.; Thompson, M. O.; Ober, C. K. Ultrafast Self-Assembly of Sub-10 Nm Block Copolymer Nanostructures by Solvent-Free High-Temperature Laser Annealing. ACS Appl. Mater. Interfaces 2017, 9, 31317-31324.

(39) Nowak, S. R.; Yager, K. G. Photothermally Directed Assembly of Block Copolymers. Adv. Mater. Interfaces 2020, 7, 1901679.

(40) Reiter, G.; Hamieh, M.; Damman, P.; Sclavons, S.; Gabriele, S.; Vilmin, T.; Raphäl, E. Residual Stresses in Thin Polymer Films Cause Rupture and Dominate Early Stages of Dewetting. Nat. Mater. 2005, 4, 754-758.

(41) Carroll, G. T.; Sojka, M. E.; Lei, X.; Turro, N. J.; Koberstein, J. T. Photoactive Additives for Cross-Linking Polymer Films: Inhibition of Dewetting in Thin Polymer Films. Langmuir 2006, 22, 7748-7754.

(42) Akhrass, S. A.; Ostaci, R. V.; Grohens, Y.; Drockenmuller, E.; Reiter, G. Influence of Progressive Cross-Linking on Dewetting of Polystyrene Thin Films. Langmuir 2008, 24, 1884-1890.

(43) Telford, A. M.; Thickett, S. C.; James, M.; Neto, C. Competition between Dewetting and Cross-Linking in Poly $(\mathrm{N}$ Vinylpyrrolidone)/Polystyrene Bilayer Films. Langmuir 2011, 27, 14207-14217.

(44) Ito, H. Chemical Amplification Resists for Microlithography. In Advances in Polymer Science; Springer: Berlin, Heidelberg, 2005; Vol. 172, pp 37-245.

(45) Shirai, M.; Okamura, H. I-Line Sensitive Photoacid Generators for UV Curing. Prog. Org. Coating 2009, 64, 175-181. 
(46) Ruiz, R.; Sandstrom, R. L.; Black, C. T. Induced Orientational Order in Symmetric Diblock Copolymer Thin Films. Adv. Mater. 2007, 19, 587-591.

(47) Li, W.; Nealey, P. F.; de Pablo, J. J.; Müller, M. Defect Removal in the Course of Directed Self-Assembly Is Facilitated in the Vicinity of the Order-Disorder Transition. Phys. Rev. Lett. 2014, 113, 168301.

(48) Rodríguez-Hernández, J. Wrinkled Interfaces: Taking Advantage of Surface Instabilities to Pattern Polymer Surfaces. Prog. Polym. Sci. 2015, 42, 1-41.

(49) Croon, J. A.; Storms, G.; Winkelmeier, S.; Pollentier, I.; Ercken, M.; Decoutere, S.; Sansen, W.; Maes, H. E. Line Edge Roughness: Characterization, Modeling and Impact on Device Behavior. In Digest. International Electron Devices Meeting; IEEE, 2002; pp 307-310.

(50) Percin, G.; Khuri-Yakub, B. T. Photoresist Deposition without Spinning. IEEE Trans. Semicond. Manuf. 2003, 16, 452-459.

(51) Sanders, D. P. Advances in Patterning Materials for $193 \mathrm{Nm}$ Immersion Lithography. Chem. Rev. 2010, 110, 321-360.

(52) Bordonaro, G. J. DUV Photolithography and Materials. In Encyclopedia of Nanotechnology; Bhushan, B., Ed.; Springer Netherlands: Dordrecht, 2012; pp 590-604.

(53) Li, L.; Liu, X.; Pal, S.; Wang, S.; Ober, C. K.; Giannelis, E. P. Extreme Ultraviolet Resist Materials for Sub-7 Nm Patterning. Chem. Soc. Rev. 2017, 46, 4855-4866.

(54) Mojarad, N.; Gobrecht, J.; Ekinci, Y. Beyond EUV lithography: a comparative study of efficient photoresists' performance. Sci. Rep. 2015, 5, 9235.

(55) Suh, K. Y.; Lee, H. H. Capillary Force Lithography: Large-Area Patterning, Self-Organization, and Anisotropic Dewetting. Adv. Funct. Mater. 2002, 12, 405-413.

(56) Kargupta, K.; Sharma, A. Templating of Thin Films Induced by Dewetting on Patterned Surfaces. Phys. Rev. Lett. 2001, 86, 45364539.

(57) Suh, K. Y.; Park, J.; Lee, H. H. Controlled Polymer Dewetting by Physical Confinement. J. Chem. Phys. 2002, 116, 7714-7718.

(58) Bandyopadhyay, D.; Singh, G.; Becker, M. L.; Karim, A. Capillary Wave Confinement-Induced Stabilization of Polymer Films. ACS Appl. Mater. Interfaces 2013, 5, 4006-4010.

(59) Jung, Y. S.; Ross, C. a. Orientation-Controlled Self-Assembled Nanolithography Using a Polystyrene-Polydimethylsiloxane Block Copolymer. Nano Lett. 2007, 7, 2046-2050.

(60) Bai, W.; Gadelrab, K.; Alexander-Katz, A.; Ross, C. A. Perpendicular Block Copolymer Microdomains in High Aspect Ratio Templates. Nano Lett. 2015, 15, 6901-6908.

(61) Han, E.; Kang, H.; Liu, C.-C.; Nealey, P. F.; Gopalan, P. Graphoepitaxial Assembly of Symmetric Block Copolymers on Weakly Preferential Substrates. Adv. Mater. 2010, 22, 4325-4329.

(62) Stefik, M.; Guldin, S.; Vignolini, S.; Wiesner, U.; Steiner, U. Block Copolymer Self-Assembly for Nanophotonics. Chem. Soc. Rev. 2015, 44, 5076-5091.

(63) Kwak, J.; Mishra, A. K.; Lee, J.; Lee, K. S.; Choi, C.; Maiti, S.; Kim, M.; Kim, J. K. Fabrication of Sub-3 Nm Feature Size Based on Block Copolymer Self-Assembly for Next-Generation Nanolithography. Macromolecules 2017, 50, 6813-6818.

(64) Ross, C. A.; Berggren, K. K.; Cheng, J. Y.; Jung, Y. S.; Chang, J.B. Three-Dimensional Nanofabrication by Block Copolymer SelfAssembly. Adv. Mater. 2014, 26, 4386-4396.

(65) Demazy, N.; Cummins, C.; Aissou, K.; Fleury, G. Non-Native Block Copolymer Thin Film Nanostructures Derived from Iterative Self-Assembly Processes. Adv. Mater. Interfaces 2020, 7, 1901747.

(66) Kim, E.; Shin, C.; Ahn, H.; Ryu, D. Y.; Bang, J.; Hawker, C. J.; Russell, T. P. Size Control and Registration of Nano-Structured Thin Films by Cross-Linkable Units. Soft Matter 2008, 4, 475-479.

(67) Hu, H.; Rangou, S.; Kim, M.; Gopalan, P.; Filiz, V.; Avgeropoulos, A.; Osuji, C. O. Continuous Equilibrated Growth of Ordered Block Copolymer Thin Films by Electrospray Deposition. ACS Nano 2013, 7, 2960-2970.

(68) Rahman, A.; Majewski, P. W.; Doerk, G.; Black, C. T.; Yager, K. G. Non-Native Three-Dimensional Block Copolymer Morphologies. Nat. Commun. 2016, 7, 13988.
(69) Jung, H.; Hwang, D.; Kim, E.; Kim, B.-J.; Lee, W. B.; Poelma, J E.; Kim, J.; Hawker, C. J.; Huh, J.; Ryu, D. Y.; Bang, J. ThreeDimensional Multilayered Nanostructures with Controlled Orientation of Microdomains from Cross-Linkable Block Copolymers. ACS Nano 2011, 5, 6164-6173.

(70) Jiang, Z. GIXSGUI: a MATLAB toolbox for grazing-incidence $\mathrm{X}$-ray scattering data visualization and reduction, and indexing of buried three-dimensional periodic nanostructured films. J. Appl. Crystallogr. 2015, 48, 917-926. 